\title{
(Broken) Gauge Symmetries and Constraints in Regge Calculus
}

\author{
Benjamin Bahr ${ }^{1}$ and Bianca Dittrich ${ }^{2}$ \\ ${ }^{1}$ DAMTP, University of Cambridge, \\ Wilberforce Road, Cambridge CB3 0WA, UK \\ ${ }^{2}$ MPI f. Gravitational Physics, Albert Einstein Institute, \\ Am Mühlenberg 1, D-14476 Potsdam, Germany \\ and \\ Institute for Theoretical Physics, Utrecht University, \\ Leuvenlaan 4, NL-3584 CE Utrecht, The Netherlands
}

Preprint AEI-2009-044

\begin{abstract}
We will examine the issue of diffeomorphism symmetry in simplicial models of (quantum) gravity, in particular for Regge calculus. We find that for a solution with curvature there do not exist exact gauge symmetries on the discrete level. Furthermore we derive a canonical formulation that exactly matches the dynamics and hence symmetries of the covariant picture. In this canonical formulation broken symmetries lead to the replacements of constraints by so-called pseudo constraints. These considerations should be taken into account in attempts to connect spin foam models, based on the Regge action, with canonical loop quantum gravity, which aims at implementing proper constraints.

We will argue that the long standing problem of finding a consistent constraint algebra for discretized gravity theories is equivalent to the problem of finding an action with exact diffeomorphism symmetries. Finally we will analyze different limits in which the pseudo constraints might turn into proper constraints. This could be helpful to infer alternative discretization schemes in which the symmetries are not broken.
\end{abstract}

\section{Introduction}

In quantizing a given theory its symmetries play a crucial role. The question whether symmetries of the classical theory have also a representation in the quantum theory can have a drastic influence on the properties of the resulting quantum theory.

For general relativity the symmetry in question is diffeomorphism invariance. As so far there is no satisfactory model of quantum gravity yet, also the fate of diffeomorphism invariance in a quantum theory of gravity is open.

Nevertheless a successful implementation of diffeomorphism invariance into quantum gravity models could ensure the correct semi-classical limit and moreover help to resolve quantization ambiguities (see for instance [1]), that could otherwise render the models unpredictive. It is therefore important to discuss notions of diffeomorphism symmetries in the models at hand.

A particular class of models, for instance Regge quantum calculus [2, 3], spin foam models [4], (causal) dynamical triangulations [5], use discretizations of the underlying spacetime manifold as a regulator in order to define the specific model, i.e. a strategy how to perform the path integral. In particular in spin foam models the Regge action appears in a semi-classical limit [6]. We will therefore concentrate on the discussion of symmetries in Regge calculus.

The main question regarding diffeomorphism invariance for such discretized models is whether a notion of exact diffeomorphism invariance can be found for the discrete model, or whether 
exact diffeomorphism invariance can arise only in a continuum limit (alternatively in a sum over triangulations, that is all possible ways of discretizations). A notion of exact diffeomorphism invariance directly on the discrete level would simplify very much the process of defining the theory, for instance in choosing the path integral measure (which then has to be diffeomorphism invariant). Furthermore, as we will show in this paper, with such a notion it should be possible to find a canonical formulation for discrete gravity models, with a closed, i.e. consistent constraint algebra. This is the main problem for canonical or Hamiltonian lattice gravity models [7, 8].

In this work we will show, that for models based on the Regge action (in 4d), diffeomorphism symmetry is generically broken. This is contrary to expectations voiced in the literature [9]. Nevertheless there are many arguments that diffeomorphism invariance will be restored in the continuum limit [10, 11, 12, and also our results will support this view.

These results do not exclude that discrete models with diffeomorphism symmetry can be constructed. Indeed, the definition of symmetry that we apply, depends crucially on the dynamics of the model, as defined by the action, in this case the Regge action. Other actions might exist, that exhibit exact diffeomorphism symmetry. We will discuss such instances of different actions for the same system with exact and broken symmetries respectively for $3 \mathrm{~d}$ Regge calculus with cosmological constant in section 6 and toy models in section 8 .

A long standing problem is the construction of a consistent discretized canonical model for gravity and a representation of diffeomorphism in such a model, see for instance [13, 14]. In a canonical formalism gauge symmetries are reflected in constraints on the canonical data, that also serve as generators for these gauge transformations. As diffeomorphisms also include transformations of the time coordinate, general relativity is a so called totally constrained system. That is the Hamiltonian, the generator for the dynamics of the system, is a combination of constraints. Hence the constraint algebra is of central importance in order to have a consistent dynamics.

Often canonical lattice models are defined by discretizing the constraints of the continuum theory. A typical problem in such discretized models is that the constraint algebra is not closed. This leads to severe problems for the quantization. According to the Dirac program constraints resulting from gauge symmetries have to be quantized and to be imposed onto the quantum states. That is however only possible if the constraint algebra is closed, or in other words anomaly free. Here the problem is already on the classical level, i.e. one has to face classical anomalies. Although different approaches exist to circumvent this problem [15, 16, 17], it might be quite hard in these approaches to keep classical and quantum anomalies and ambiguities under control. This would be easier if we could construct discretized models with a closed algebra. Here we will show that this problem can be seen to be equivalent to finding an action, i.e. a covariant model with exact diffeomorphism invariance. If the action displays exact gauge invariance we will find constraints generating the gauge transformations in the canonical formalism. For actions with broken symmetries we will find a different picture: Instead of exact constraints - that is relations imposed by the dynamics that happen to involve data of only one time step, we will find pseudo constraints, that is dynamical relations which show a (weak) dependence also on the data on the next time step. This dependence can be interpreted as a dependence on the Lagrange multipliers lapse and shift. This allows to solve the pseudo constraints for lapse and shift. Hence gauge freedom is lost (as it is indeed broken on the covariant level) and there are no constraints left on the canonical data - a picture that was advertised in the consistent discretization program [18]. We will however argue that - as one expects gauge symmetries to be restored in the continuum limit - it might be more promising to keep the pseudo constraints. Indeed, data leading to solutions with a small discretization scale, are concentrated on a 'thickened constraint hypersurface'. In the quantum theory the connection between covariant models with exact gauge symmetry and canonical models with 
constraints is, that the path integral acts as a projector onto the space of states satisfying the constraints [19]. For systems with slightly broken symmetries one would expect instead of an exact projector an approximate implementation of the constraints, similar to a delta function versus a Gaussian.

The advantage of the technique used in this work is that the dynamics as defined by the covariant equations of motion and the canonical time evolution equations coincide and hence also display the same amount of gauge symmetry. These methods are in particular important for attempts to establish a closer connection between spin foam models and (canonical) loop quantum gravity. In the latter dynamics is based on exact constraints whereas in the former we expect diffeomorphism symmetry to be broken (as it is broken for the Regge action and the Regge action appears in a semi-classical analysis [6] of current spin foam models). With this different handling of symmetries by the two models the dynamics as defined by these models very likely also differs - at least on the discrete level.

In the next section we will discuss the notion of gauge symmetries we are going to apply and how to test for the existence of these symmetries. Next we discuss an evolution scheme for Regge calculus, the so-called tent moves, which we are going to need for the construction of a solution with curvature as well as for developing a canonical formulation. We construct such solutions in section 5 and determine the eigenvalues of the Hessian for these solutions. In section 6 we discuss a canonical formulation for $3 \mathrm{~d}$ discretized gravity with cosmological constant using discrete actions with exact and with broken symmetries. Furthermore we analyze limits in which the broken symmetries might turn into exact ones. We will show in section 7 that if we have exact gauge symmetries in the action then we will find proper constraints on the discrete canonical data. Section 8 provides another class of simple examples of discretized theories with broken gauge symmetries. For these class of theories however one can always define a discretization with exact gauge symmetries. We will close with a discussion in section 9. The appendices A and $\mathrm{B}$ contain a description of two physically different solutions with the same boundary data and formulas for geometrical quantities of simplices that we need in section 6 respectively.

\section{Definition of gauge symmetry}

In this section we will shortly describe the notion of diffeomorphism symmetry that we are going to apply.

First of all we will consider continuously parametrized gauge symmetries. (As discussed in [20] there might also exist notions which are completely based on discrete transformations, such as a change of triangulation. We will comment shortly on a possible relation to continuum symmetries in the discussion section.) In the continuum such gauge symmetries lead to a continuous family of solutions to the equations of motions (with fixed boundary values for the variables), instead of just having one solution. We will apply the same definition to discrete models. That is we will speak of an (exact) gauge symmetry if the boundary value problem displays non-uniqueness of solutions, moreover this non-uniqueness should be parametrizable in a continuous way.

As solutions to the equations of motions are extrema of the actions, this means that the action is constant in some directions exactly at these extrema. As already discussed in [20] it is important to check that these constant directions also persist at the extrema of the action, that is at solutions. (Away from solutions any direction perpendicular to the gradient is a constant direction.) This means that it is not sufficient to identify (possibly configuration dependent) transformations that leave the action invariant as these transformations might act trivially on solutions. In this case we will still have uniqueness of solutions.

If there is a continuous parameter set of solutions then there exist directions at these solutions 
in which the action is constant and also the first derivatives of the action are constant (and equal to zero). Hence in this case the Hessian, the matrix of second derivatives, of the action, will have null eigenvectors. This criterion is therefore a necessary condition for a gauge symmetry.

Note that different solutions might have gauge orbits of different size. There might be theories with solutions with gauge symmetries and solutions without gauge symmetries. As we will see this is the case for $4 \mathrm{~d}$ Regge calculus. There, flat solutions display gauge symmetries [21, 22, 23]. Vertices of the triangulation supporting this flat solution can be translated (in four directions) without changing the flatness of the solutions. In contrast, for solutions with curvature (and moreover without any flat vertices 1 ) we do not find gauge symmetries. Hence we have a mixture of exact gauge symmetries (for flat vertices) and broken symmetries.

We will show explicitly that the criterion of vanishing eigenvalues of the Hessian (evaluated at solutions) is violated for a $4 \mathrm{~d}$ Regge solution with curvature. But this example will also show that gauge invariance is only slightly broken, hence we can speak of approximate gauge invariance. Namely we will see that some of the eigenvalues of the Hessian (per vertex) are very small as compared to the rest of the eigenvalues. Moreover in approaching the flat solution these eigenvalues go to zero (quadratically in the curvature). Hence analyzing the eigenvalues of the Hessian is a precise tool to discuss approximate gauge symmetries. This might be helpful in order to construct actions with an exact gauge invariance.

In section 7 we will furthermore see that the criterion of null eigenvalues of the Hessian is related to the appearance (or non-appearance) of constraints in a canonical formulation of the theory.

\section{Tent moves}

Tent moves are a way of defining a discrete time evolution for Regge calculus, which has first been described in 24], and used for several works in Regge calculus [12. It is a way to evolve a triangulated hypersurface locally, such that the triangulation (that is the adjacency relations) of the resulting new hypersurfaces does not change. These tent moves are a very convenient tool to define a canonical formalism [20] for Regge calculus. Implementing some ideas from discrete numerical integration [25] or 'consistent discretization' [18] the dynamics defined by the canonical and covariant formulations will exactly coincide and therefore also the gauge symmetries in these formulations.

Consider a $(d-1)$-dimensional triangulation $\Sigma$, which can be thought of as a triangulated Cauchy hypersurface. Pick a vertex $v$ in the triangulation and define a new vertex $v^{*}$ lying in the 'future' of $v$, and connect both vertices with an edge. Denote all other vertices in $\Sigma$ that $v$ is connected to by $1, \ldots, n$. Connect also $v^{*}$ to the $1, \ldots, n$ by edges. Furthermore we will have a simplex $v^{*} i j(k)$ (with vertices $v^{*}, i, j$ in $3 \mathrm{~d}$ and $4 \mathrm{~d}$ and $v^{*}, i, j, k$ in $4 \mathrm{~d}$ ) in the evolved hypersurface $\Sigma^{*}$ for every simplex vij $(k)$ in $\Sigma$. Hence the triangulations of the two Cauchy surfaces are the same. The evolution can be thought of as gluing a certain piece of $d$-dimensional triangulation onto the hypersurface. This $d$-dimensional triangulation consists of simplices $v v^{*} i j(k)$ for every simplex $v i j(k)$ in $\Sigma$ in addition to simplices in the boundary coinciding with either $\Sigma, \Sigma^{*}$ or both. The edge connecting $v$ and $v^{*}$ is called the "tent-pole".

Note that each tent move can be generated by a sequence of Pachner moves applied to the Cauchy surfaces. For $d=3$, an $n$-valent tent move is the result of a $1-3$-move, followed by $(n-2) 2-2$ moves, and finally a $3-1$ move. By applying tent moves to various vertices after another, one can build up a large $d$-dimensional triangulation.

There are several advantages of this description:

\footnotetext{
${ }^{1}$ That is, all triangles adjacent to these vertices have vanishing deficit angles.
} 


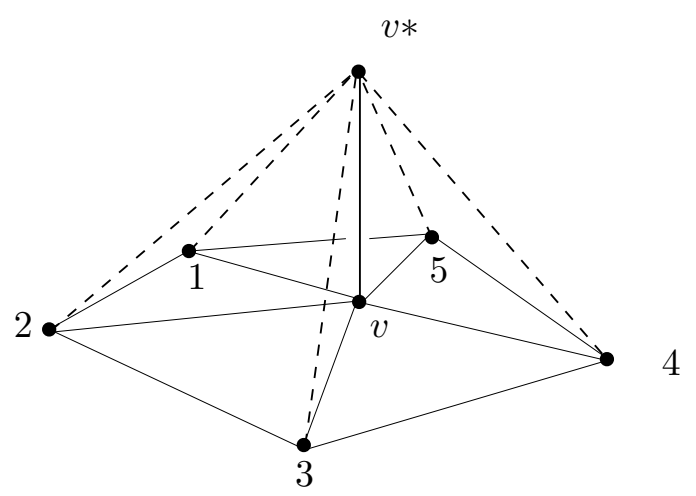

Figure 1: A 5-valent tent move at the vertex $v$.

- The evolution is local, in the sense that only the triangulation containing the vertex $v$ (called the "star of $v$ ") is evolved, while the rest of the triangulation remains untouched.

- As a result, one can evolve a collection of vertices independently of each other if neither of the vertices can be connected to any other of the collection. This has in particular been implemented in numerical applications [12].

- The tent moves are particularly useful for an investigation of Regge calculus in a canonical language, since all Cauchy hypersurfaces that are produced in each step are isomorphic (as simplicial complexes). Therefore, in each step the number of canonical variables on the hypersurface remains unchanged. This is not true in an arbitrary triangulation, which makes the canonical analysis harder, and is the main reason why we consider tent moves in this work. Furthermore for the analysis of a tent move we need to consider only a small number of equations as opposed to a scheme in which all vertices are evolved at once.

- The choice of which vertices of a hypersurface to evolve can be understood as a discrete choice of lapse (to be either vanishing or non-vanishing). Also, if $v_{1}$ and $v_{2}$ are vertices in the initial triangulation that are connected by an edge, the two tent moves applied to $v_{1}$ and $v_{2}$ do not commute. If one first "evolves" $v_{1}$ and then $v_{2}$, one obtains a different ( $d$-dimensional) triangulation than first evolving $v_{2}$ and then $v_{1}$. That might serve as a starting point for a definition of a discrete notion for a hypersurface deformation algebra [20].

\subsection{Evolution equations for tent moves}

The Regge action for a $d$-dimensional triangulation $T$ with boundary $\partial T$ and interior $T^{\circ}:=$ $T \backslash \partial T$ is given [2, 26] by

$$
S_{\text {Regge }}=-\sum_{h \in T^{\circ}} V_{h} \epsilon_{h}-\sum_{h \in \partial T} V_{h} \psi_{h}+\lambda \sum_{\sigma \in T} V_{\sigma}
$$

where the $h$ are the $d-2$ dimensional subsimplices (sometimes called "hinges") of $T$ and $\sigma$ are the top-dimensional simplices of the triangulation. $V_{h}$ and $V_{\sigma}$ denote the volume of the hinge $h$ and of the simplex $\sigma$ respectively. The deficit angles $\epsilon_{h}$ and exterior angles $\psi_{h}$ are given by

$$
\begin{aligned}
\epsilon_{h}=2 \pi-\sum_{\sigma \subset h} \theta_{h}^{\sigma} \quad \text { for } h \in T^{\circ} \\
\psi_{h}=\pi-\sum_{\sigma \subset h} \theta_{h}^{\sigma} \quad \text { for } h \in \partial T,
\end{aligned}
$$


and in both cases the sum ranges over all $d$-dimensional simplices $\sigma$ which contain $h$, and $\theta_{h}^{\sigma}$ is the interior dihedral angle in the simplex $\sigma$ between the two $(d-1)$-dimensional subsimplices that meet at $h \subset \sigma$.

Note that because of the boundary term in (3.1) the action is additive if we glue two pieces of triangulations together.

The equations of motion are obtained by varying the action (3.1) with respect to the lengths $l_{e}$ of the edges in $T^{\circ}$. The boundary term in (3.1) ensures also that, for all edges $e \in T^{\circ}$ the Regge equations read

$$
-\sum_{h \supset e} \frac{\partial V_{h}}{\partial l_{e}} \epsilon_{h}+\lambda \sum_{\sigma \supset e} \frac{\partial V_{\sigma}}{\partial l_{e}}=0 .
$$

The variation of the deficit angles appearing in the action vanishes because of the Schläfli identity, see appendix B.

Consider a tent move for an $n$-valent vertex $v$ in the boundary $\Sigma$ of a $d$-dimensional triangulation $T$. After the tent move is performed we will have a new triangulation $T^{*}$ with $(n+1)$ new inner edges, namely the edges $v i, i=1, \ldots n$ adjacent in $\Sigma$ to the vertex $v$ and the tent pole $v v^{*}$. We will denote by $S_{T}, S_{T^{*}}$ and $S_{*}$ the action (with boundary terms) of the original triangulation $T$, the new triangulation $T^{*}$ and the piece of triangulation added in the tent move, so that we have $S_{T^{*}}=S_{T}+S_{*}$. The equations for the new inner edges can then be written as

$$
\begin{aligned}
\frac{\partial S_{T}}{\partial l_{v i}}+\frac{\partial S_{*}}{\partial l_{v i}} & =0 \\
\frac{\partial S_{*}}{\partial l_{v v^{*}}} & =0
\end{aligned} .
$$

With the definitions

$$
\begin{aligned}
p_{v i}^{-}:=\frac{\partial S_{T}}{\partial l_{v i}} & p_{v i}^{+}:=-\frac{\partial S_{*}}{\partial l_{v i}} \\
p_{v v^{*}}^{-}:=\frac{\partial S_{T}}{\partial l_{v v^{*}}} & p_{v v *}^{+}:=-\frac{\partial S_{*}}{\partial l_{v v^{*}}} \\
p_{v^{*} i}^{-}:=\frac{\partial S_{*}}{\partial l_{v^{*} i}} & p_{v^{*} v^{* *}}^{+}:=-\frac{\partial S_{*}}{\partial l_{v^{*} v^{* *}}}
\end{aligned}
$$

the equations of motion (3.4) are now given by

$$
p_{v i}^{+}=p_{v i}^{-} \quad p_{v v^{*}}^{+}=p_{v v^{*}}^{-}=0
$$

that is the momenta $p^{-}$and $p^{+}$defined as derivatives of the actions associated to the two pieces of the triangulation $T^{*}$ have to coincide. Therefore we will omit the superindices,+- . With the second and third line in (3.5) we use the action of the added piece as a generating function of first kind to define a canonical transformation from the canonical variables $\left(l_{v i}, l_{v v^{*}}, p_{v i}, p_{v v^{*}}\right)$ to a set of new canonical variables $\left(l_{v^{*}}, l_{v^{*} v^{* *}}, p_{v^{*}}, p_{v^{*} v^{* *}}\right)$ (where we introduced a fiducial vertex $v^{* *}$ which can be thought of as the vertex added in a second tent move). Here we see that the length of the tent pole has a special status as its conjugated momentum is constrained to vanish. Hence this variable is not fully dynamical. This corresponds to an analogous result in the continuum where in the canonical analysis the momenta conjugated to lapse and sift are constrained to vanish.

Equations (3.5) are just a reformulation of the equations of motion in canonical language by using the action $S_{*}$ as a generating function for a canonical transformation. This allows us to obtain a canonical formalism which reflects exactly the dynamics of the covariant formulation. 
Other attempts to define a canonical framework for Regge calculus usually involve changing the dynamical set up [7]. Therefore gauge symmetries of the covariant formulation might not be reflected properly in the canonical formulation.

The advantage in the formulation used here is that the dynamics defined in the canonical formalism is the same as the covariant dynamics. Gauge symmetries of certain or all solutions should therefore have repercussions for the canonical formulation.

\section{Remarks on discrete ambiguities of solutions to the Regge equations}

Given a boundary value problem the question arises whether the solutions are unique. Gauge symmetries in the form discussed here lead to a continuous family of non-unique solutions. In addition there might be discrete ambiguities.

These also appear in Regge calculus, as for instance reported in [27]. In our investigations we noticed ambiguities already for the smallest boundary value problem, namely a triangulation with only one inner edge. Such ambiguities are common to discretizations of continuum theories. Typically there is only one solution that is useful in a continuum limit whereas the others can be seen as discretization artifacts. Nevertheless the question of discrete ambiguities should be explored in more detail, as these might influence the quantum theory.

Some of the ambiguities arise because of the following: If we consider for instance a closed $4 \mathrm{~d}$ ball with its $3 \mathrm{~d}$ triangulated boundary, then pieces of this $3 \mathrm{~d}$ triangulation might stick inwardly or outwardly. Similarly if we solve the tent pole equation (the second equation in (3.4)) for the length of the tent pole then typically there is one solution which is forward pointing, i.e. a (bigger) tent is built on a smaller tent and another solution, that is backward pointing, i.e. resulting in the tips of two tents in opposite direction. Here we will always select the forward pointing direction for the canonical analysis.

In the appendix $\mathrm{A}$ we will discuss another kind of ambiguity (arising by setting the prefactor $\frac{\partial V_{h}}{\partial l_{e}}$ in the equations of motion (3.3) to zero). There we construct a boundary value problem which allows for a flat and a curved solution (with the same boundary data). The curved solution has however very high curvature and one reason for its appearance seems to be the highly symmetric situation. It can therefore considered to be a discretization artifact.

\section{Construction of a Regge solution via tent moves}

Here we will describe shortly how to find numerically a small Regge solution with curvature using the tent moves. The evolution of four-valent vertices should lead to flat solution:2: indeed such solutions can be constructed by subdividing accordingly a flat 4-simplex.

Therefore the simplest case to consider is the evolution of a five-valent vertex. To this end we have to define the three-dimensional triangulation around the vertex $v$, we want to evolve, more concretely the three-dimensional star of $v$. As $v$ is five-valent we have five further vertices which we will denote by $1, \ldots, 5$. We will assume that we have six tetrahedra with vertices

$$
v 124, \quad v 134, \quad v 234, \quad v 125, \quad, v 135, \quad v 235 \quad .
$$

Accordingly we will have nine triangles of the form $v i j$ with $i, j=1, \ldots 5$ in this triangulation, five edges of the form $v i$ and nine edges of the form $i j$ (all possible ordered combinations of $i, j \in\{1, \ldots 5\}$ with the exception 45$)$.

\footnotetext{
${ }^{2}$ Apart from the discrete ambiguity described in the appendix.
} 


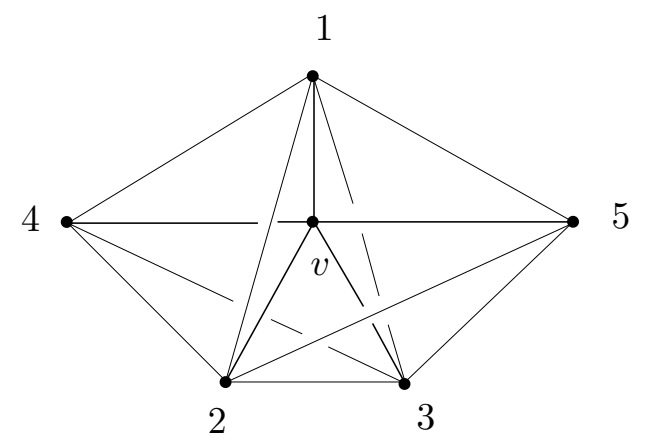

Figure 2: The 3d boundary of the tent move triangulation.

To simplify the calculations even further we will assume that all edges $i j$ have the same length $l_{i j}=s$. As the Regge vacuum equations are invariant under a global rescaling we will set $s=1$. We will also assume that $l_{v i}=a, i=1,2,3$ are equal to each other as well as $l_{v 4}=l_{v 5}=b$. Hence we have to deal with two dynamical configuration variables $a$ and $b$. By $a^{n}, b^{n}$ we will denote the values of these variables at the time step $n$. Together with the lengths of the tent poles $t^{n}$ these will be also the free variables in the action. (Varying the action with respect to all the inner edge length and then looking for solutions with $l_{v i}=a, l_{v \kappa}=b$ is equivalent to using this reduction in the action and varying with respect to $a, b$ and the lengths of the tent poles. In this sense we will consider a symmetry reduced action.)

The 4-simplices involved in the tent move are all of the same type $v^{0} v^{1} i j \kappa$ where $v^{0}, v^{1}$ denote the two vertices of the tent pole (at time steps $n=0, n=1$ respectively), $i, j$ take values in $1,2,3$ and $\kappa$ in 4,5 . We will denote by

$\theta_{a}^{0}, A_{a}^{0}$ the dihedral angle and the area of the triangle $v^{0} i j$,

$\theta_{b}^{0}, A_{b}^{0}$ the dihedral angle and the area of the triangle $v^{0} i \kappa$,

$\theta_{t}^{a}, A_{t}^{a}$ the dihedral angle and the area of the triangle $v^{0} v^{1} i$,

$\theta_{t}^{b}, A_{t}^{b}$ the dihedral angle and the area of the triangle $v^{0} v^{1} \kappa$,

$\theta_{a}^{1}, A_{a}^{1}$ the dihedral angle and the area of the triangle $v^{1} i j$,

$\theta_{b}^{1}, A_{b}^{1}$ the dihedral angle and the area of the triangle $v^{1} i \kappa$ respectively .

The canonical equations of motion determining $a^{1}, b^{1}$ and $t^{0}$ given initial values $a^{0}, b^{0}$ and $p_{a}^{0}$ and $p_{b}^{0}$ are given by

$$
\begin{aligned}
0 & =3 \frac{\partial A_{t}^{a}}{\partial t^{0}}\left(2 \pi-4 \theta_{t}^{a}\right)+2 \frac{\partial A_{t}^{b}}{\partial t^{0}}\left(2 \pi-3 \theta_{t}^{b}\right) \\
p_{0}^{a} & =\frac{\partial A_{a}^{0}}{\partial a^{0}}\left(\pi-2 \theta_{a}^{0}\right)+\frac{\partial A_{b}^{0}}{\partial a^{0}}\left(\pi-2 \theta_{b}^{0}\right)+\frac{\partial A_{t}^{a}}{\partial a^{0}}\left(2 \pi-4 \theta_{t}^{a}\right) \\
p_{0}^{b} & =3 \frac{\partial A_{b}^{0}}{\partial b^{0}}\left(\pi-2 \theta_{b}^{0}\right)+\frac{\partial A_{t}^{b}}{\partial b^{0}}\left(2 \pi-3 \theta_{t}^{b}\right)
\end{aligned}
$$

The new momenta $p_{1}^{a}$ and $p_{1}^{b}$ are defined as

$$
\begin{aligned}
& p_{1}^{a}=-\left(\frac{\partial A_{a}^{1}}{\partial a^{1}}\left(\pi-2 \theta_{a}^{1}\right)+\frac{\partial A_{b}^{1}}{\partial a^{1}}\left(\pi-2 \theta_{b}^{1}\right)+\frac{\partial A_{t}^{a}}{\partial a^{1}}\left(2 \pi-4 \theta_{t}^{a}\right)\right) \\
& p_{1}^{b}=-\left(3 \frac{\partial A_{b}^{1}}{\partial b^{1}}\left(\pi-2 \theta_{b}^{1}\right)+\frac{\partial A_{t}^{b}}{\partial b^{1}}\left(2 \pi-3 \theta_{t}^{b}\right)\right)
\end{aligned}
$$

To construct a solution with an inner vertex $v^{1}$ we have to consider at least two consecutive tent moves. We will proceed in the following way. First we will start with some initial data 
$\left(a^{0}, b^{0}, a^{1}, b^{1}\right)$ and use equation (5.2) to find the length of the tent pole $t^{0}$. Given these five length we can determine the momenta $\left(p_{a}^{1}, p_{b}^{2}\right)$ through equations (5.4). Now we have a full set of initial canonical data and can use all three equations (5.25.3) to find $t^{1}, a^{2}, b^{2}$. We will end up with a Regge solution with inner vertex $v^{1}$. In the end we have to evaluate the matrix of second derivatives of the action with respect to $\left(a^{1}, b^{1}, t^{0}, t^{1}\right)$ on this solution and determine its eigenvalues.

However if one attempts to solve the equations (5.25.3) for $t^{1}, a^{2}, b^{2}$ numerically one will typically encounter the difficulty that standard numerical (iterative) procedures do not converge. This is due to the fact that there is at least an approximate gauge invariance (corresponding to choosing the lapse at the vertex). So if we reformulate this problem as finding the extrema of the action, we will have the difficulty that there exist a direction in which the action is almost constant and the extremum along this direction is difficult to locate. The corresponding small eigenvalue of the Hessian leads to convergence problems of the numerical procedures.

This difficulty is usually circumvented by for instance (gauge) fixing the value for $t^{1}$ and ignoring one of the equations, for instance (5.2) coming from the variation with respect to $t^{1}$ [12]. One then hopes that this equation is satisfied up to a certain small error. This error is basically determined by the value of the smallest eigenvalue in the Hessian.

To obtain a solution to a predetermined precision $\delta$ we can guess a value for $t^{1}$, solve the second and third equations for $a^{2}$ and $b^{2}$ and then evaluate the right hand side of equation (5.2). If this error $E\left(t_{1}\right)$ is larger than $\delta$ we have to start again with another value of $t^{1}$. This procedure can be systemized by starting with two values for $t^{1}$, say $A, B$ and determining $E(A), E(B)$. If the signs of $E(A)$ and $E(B)$ are different, than there is a zero in $[A, B]$ (assuming that $E$ is continuous on this interval). This zero can be determined iteratively to arbitrary precision.

We want to generate a set of solutions deviating slightly from the flat solution. Hence we first construct a flat solution and then introduce a deviation from the flat boundary data. Flat solutions can be found by starting from a given data set $\left(a^{0}, b^{0}, t_{f}^{0}\right)$ and considering the deficit angles at the triangles $v^{0} v^{1} i, i=1,2,3$ and $v^{0} v^{1} \kappa, \kappa=4,5$. Setting these to zero gives two equations and we can find values $a_{f}^{1}\left(a^{0}, b^{0}, t_{f}^{0}\right)$ and $b_{f}^{1}\left(a^{0}, b^{0}, t_{f}^{0}\right)$ satisfying these equations. Since we set certain edge lengths equal to each other we have in this case only one lapse degree of freedom, i.e. no shift degrees of freedom. This means that we find one flat solutions for any given value of $t_{f}^{0}$ (and $\left.a^{0}, b^{0}\right)$.

Evaluating the Hesse matrix (second derivatives of the action with respect to $\left.\left(a^{1}, b^{1}, t^{0}, t^{1}\right)\right)$ on flat solutions we will find one zero eigenvalue corresponding to the lapse degree of freedom. Hence for a solution with curvature we expect either one vanishing eigenvalue in case that the symmetry of the flat solutions persists - or at least one very small but nonvanishing eigenvalue indicating that the symmetry is broken for curved solutions.

To obtain curved solutions we start with boundary data $\left(a^{0}, b^{0}, a_{f}^{1}, b_{f}^{1}+x\right)$ deviating from the flat solution. We solve numerically for $t^{0}, a^{2}, b^{2}$ and $t^{1}$ (with error terms of the order $10^{-13}$ ).

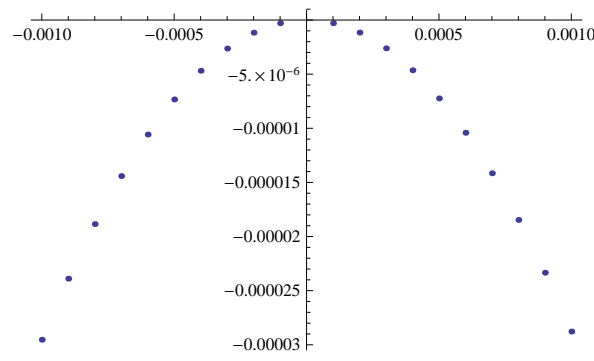

Figure 3: The lowest eigenvalue of the Hessian as a function of the deviation parameter $x$.

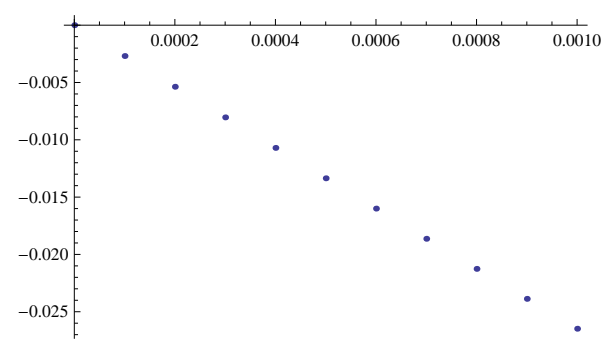

Figure 4: The deficit angle at the triangle $v^{0} v^{1} i$ as a function of the deviation parameter $x$. 
The plot in Figure 3 is for initial data generated as described above with $a^{0}=b^{0}=1$ and $a_{f}^{1}=1.16902, b_{f}^{1}=1.13356$ corresponding to the choice $t_{f}^{0}=0.2$. As can be seen from this plot, the lowest eigenvalue obtains non-zero values for solutions with curvature. Compared to the other eigenvalues the lowest eigenvalue is very small as can be seen for the set of data given in Table 1. In Figure 4 we plotted the deficit angle at one of the inner triangles as a function of the deviation parameter $x$ showing that we are indeed considering solutions with non-vanishing curvature. Furthermore we see that the lowest eigenvalue seems to grow quadratically with the curvature.

\begin{tabular}{|c|c|c|c|c|}
\hline $\mathrm{x}$ & eigenvalue 1 & eigenvalue 2 & eigenvalue 3 & eigenvalue 4 \\
\hline 0.0001 & 1678.52 & 570.127 & -394.796 & $-2.91136 \times 10^{-7}$ \\
0.001 & 1659.28 & 562.471 & -383.002 & $-2.87768 \times 10^{-5}$ \\
0.01 & 1528.00 & 509.495 & -306.545 & $-2.57318 \times 10^{-3}$ \\
\hline \hline
\end{tabular}

Table 1: Eigenvalues of the Hessian as a function of the deviation parameter $x$. The inner vertex has seven adjacent edges, but due to the symmetry of the problem, these are associated to only four variables.

This shows that for these vertices with curvature we do not have symmetries which can be associated to translating the vertex. We constructed also other examples (see appendix $\mathrm{A}$ for one other solution) where again for solutions with curvature we do not find any vanishing eigenvalues of the Hessian. This does not exclude that some curved solutions exist, which have vanishing eigenvalues - however this seems to be a rather non-generic case.

Apart from the result that the symmetries in Regge calculus are broken for vertices with curvature we want to point out that with the methods presented here we can easily check to which extent the symmetries are broken and how this scales with the curvature. The example considered here indicates that the symmetry breaking grows quadratically with the curvature. As we will see in section 6 this might allow for a canonical formalism in which we obtain proper constraints up to terms with a specific order in the curvature.

\section{Canonical analysis for 3d Regge calculus with cosmological constant}

In this section we will investigate the consequences of broken and exact gauge symmetries in the action for a canonical formalism. Here we will discuss 3d Regge calculus with and without a cosmological constant term as this example provides us with both cases, one in which the symmetries are exact and one in which they are broken. Moreover it allows us to discuss limits in which the broken symmetries may become exact. The analysis of $4 \mathrm{~d}$ Regge calculus (in which the symmetries are generically broken) will be postponed to a seperate paper [28].

The solutions of $3 \mathrm{~d}$ dimensional general relativity without a cosmological constant are locally flat. Correspondingly the $3 \mathrm{~d}$ Regge equations just require that the deficit angles, which are attached to the edges, should be vanishing. Hence every triangulation of a locally flat space is a solution and moreover there is a three-parameter gauge freedom attached to every inner vertex of the triangulation. This gauge freedom corresponds to translating an inner vertex such that the triangulation remains flat.

This gauge freedom vanishes if we consider a non-vanishing cosmological constant and use the standard Regge action with an added volume term

$$
S=-\sum_{e \in T^{\circ}} l_{e} \epsilon_{e}-\sum_{e \in \partial T} l_{e} \psi_{e}+\lambda \sum_{\tau \in T} V_{\tau}
$$


where $l_{e}, \epsilon_{e}$ are the length and the deficit angles respectively associated to the edge $e, \psi_{e}$ is the exterior angle at a boundary edge $e \in \partial T$ and $V_{\tau}$ is the volume of a tetrahedron $\tau$ [29, 20].

Indeed we can consider also in this case small $3 \mathrm{~d}$ triangulations $T$ with an inner vertex and evaluate the Hessian on a solution. In this case we used two consecutive tent moves at a three-valent vertex to construct such a small $3 \mathrm{~d}$ triangulation. The results show small but nonvanishing eigenvalues corresponding to the approximate gauge symmetries, see Figures 5 and 6 . We considered homogeneous solutions for which the three length variables at the evolved vertex coincide. The initial values are $l^{0}=1, l^{1}=1.8$ for the length variables at time step 0 and 1 , and $s=1$ for the non-dynamical edges not adjacent to either $v^{0}, v^{1}, v^{2}$. Nevertheless we can consider the Hessian with derivatives for all five variables (the three length variables and the two tent pole variables) and evaluate this Hessian on the homogeneous solution. Because of the homogeneous configuration there are two small eigenvalues coinciding which correspond to translations of the vertex in space-like directions (associated to spatial diffeomorphism constraints), that is variations for which the tent pole variables are constant. Then there is another small eigenvalue corresponding to a translation in tent pole direction (associated to a Hamiltonian constraint).

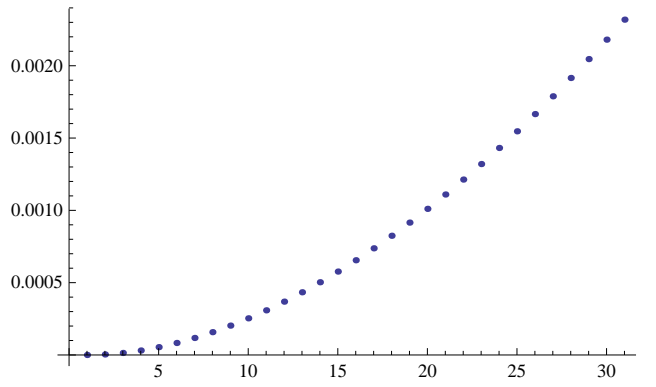

Figure 5: The eigenvalue corresponding to translations in tent-pole direction as a function of $\lambda=0.01 \times x$-axis label

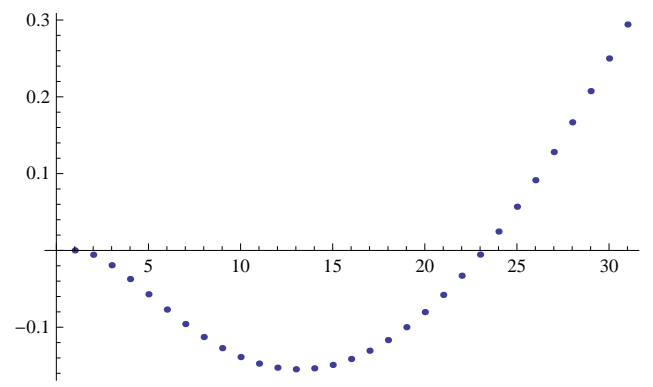

Figure 6: The eigenvalues corresponding to translations in space-like direction as a function of $\lambda=0.01 \times x$-axis label

What makes the $3 \mathrm{~d}$ case with cosmological constant interesting for us is that there is actually an action, defining a discretized dynamics, in which these gauge symmetries are exact [30]. Starting with the standard 3d Regge action we can therefore test different methods to construct an action with exact gauge symmetries and see if we obtain the same result.

In this section we will start with the $3 \mathrm{~d}$ standard Regge action and perform a canonical analysis using tent moves. Since the gauge symmetries are only approximate we will find that we do not obtain exact constraints. Rather we have to deal with pseudo constraints which depend on lapse and shift.

One could try to obtain exact constraints by considering a limit in which the length of the tent pole (and therefore lapse and shift) goes to zero. Indeed such a strategy works for discretized reparametrization invariant systems as is explained in section 8, In this case however one cannot obtain useful results. The reason is that gauge invariance is not being restored in this limit. Note that here we keep the spatial discretization scale fixed and send the discretization scale in time direction to zero. That results in almost degenerate simplices. As is also argued in [11] one cannot hope for a restoration of diffeomorphism symmetry for such degenerate simplices.

On the other hand one can consider the limit in which both the discretization scale in spatial and time directions are small. By rescaling the edge length and the cosmological constant appropriately this corresponds to small cosmological constant $\lambda$. We will see that to first order in $\lambda$ the constraints do not depend on lapse and shift. Moreover these constraints correspond to the first order constraints obtained from the alternative action with exact gauge symmetries. 


\subsection{Canonical analysis of a tent move}

We will consider the tent move evolution of a three-valent vertex $v$. We will denote the length of the edges between $v^{n}$, where the superindex $n$ denotes the time step, and the adjacent vertices $1,2,3$ by $l_{i}^{n}, i=1,2,3$. The length of the tent pole between vertices $v^{n}$ and $v^{n+1}$ is $t^{n}$. Furthermore the (non-dynamical) lengths of the edges $i j$ will be denoted by $s_{i j}$. With $S_{(n, n+1)}$ we denote the action with boundary terms for the piece of triangulation that is added by performing a tent move. In this case the added piece are three tetrahedra joined at the tent pole.

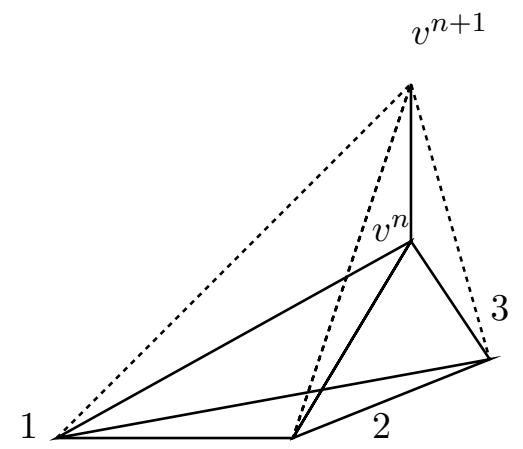

Figure 7: A tent move for a three-valent vertex.

According to (3.513.6) the canonical time evolution equations are given by

$$
\begin{aligned}
p_{i}^{n} & =-\frac{\partial S_{(n, n+1)}}{\partial l_{i}^{n}}=\psi_{i}^{n+}-\lambda \frac{\partial V_{(n, n+1)}}{\partial l_{i}^{n}} \\
p_{t}^{n} & =-\frac{\partial S_{(n, n+1)}}{\partial t^{n}}=\epsilon_{t}^{n}-\lambda \frac{\partial V_{(n, n+1)}}{\partial t^{n}} \\
p_{i}^{n+1} & =\frac{\partial S_{(n, n+1)}}{\partial l_{i}^{n+1}}=-\psi_{i}^{(n+1)-}+\lambda \frac{\partial V_{(n, n+1)}}{\partial l_{i}^{n+1}} \\
p_{t}^{n+1} & =\frac{\partial S_{(n, n+1)}}{\partial t^{n+1}}=0 .
\end{aligned}
$$

Here $\psi_{i}^{n+}$ is the exterior angle at the edge $v^{n} i$ of the triangulation between time steps $n$ and $(n+1)$ whereas we denoted with $\psi^{(n+1)-}$ the exterior angle at the edge $v^{n+1} i$ of the same piece of triangulation. $\epsilon_{t}^{n}$ is the deficit angle at the tent pole $v^{n} v^{n+1}$ and $V_{(n, n+1)}$ the volume of the triangulation between the time steps $n$ and $(n+1)$.

Because of the last equation in (6.2) we have that $p_{t}^{n}=0$ for all time steps $n$. We want to solve the second equation $p_{t}^{n}=0$ for $t^{n}=t^{n}\left(l_{i}^{n}, l_{i}^{n+1}\right)$ and use this solution in the first equation. For $\lambda \neq 0$ this equation however involves sums of trigonometric functions and cannot be solved explicitly. Nevertheless it is possible to make an ansatz $t^{n}={ }^{0} t^{n}+\lambda{ }^{1} t^{n}+\ldots$ and solve this equation order by order in $\lambda$.

The zeroth order gives the dynamics for vanishing cosmological constant, that is solutions are locally flat and all deficit angles have to vanish. The solution ${ }^{0} t^{n}$ to the second equation can be easily constructed by geometrical considerations, it is the length of the edge between the two tips of a double pyramid with triangular base. (In general there are two solutions, one in which the tips of the pyramid are in opposite direction and one in which the tips are in the same direction. We will use the latter case.) Using this solution in the first equation in (6.2) (with $\lambda=0$ ) one will find that the dependency on the length $l_{i}^{n+1}$ drops out and that the momenta are constrained to be

$$
p_{i}^{n}=-\pi+\theta_{v^{n} i}\left(l_{i}^{n}, s_{i j}\right)
$$


where with $\theta_{v^{n}} i\left(l_{i}^{n}, s_{i j}\right)$ we denote the dihedral angle at the edge $v^{n} i$ of a tetrahedron with edge lengths $l_{i}^{n}$ and $s_{i j}$ and with vertices $v^{n}, 1,2,3$.. Indeed since the dynamics of the theory just results in flat space the intrinsic and extrinsic geometry of the hypersurface resulting from the tent move evolution will be just the same as that of the boundary of a flat tetrahedron embedded in flat $3 \mathrm{~d}$ space. The constraints (6.3) just express this geometrical fact.

If we instead use the solution ${ }^{0} t^{n}={ }^{0} t^{n}\left(l_{i}^{n}, l_{i}^{n+1}\right)$ in the third equation of (6.2) , which defines the momenta $p_{i}^{n+1}$ at the time step $(n+1)$ we will find that the dependence on the length variables $l_{i}^{n}$ drops out and that the constraints are preserved by time evolution 3

$$
p_{i}^{n+1}=-\pi+\theta_{v^{n+1} i}\left(l_{i}^{n+1}, s_{i j}\right)
$$

To summarize, for $\lambda=0$, the canonical evolution equations (6.2) do not determine uniquely the evolution for the lengths $l_{i}^{n+1}$ and the momenta $p_{i}^{n+1}$, they rather result in three constraints determining the momenta $p_{i}^{n}$ and $p_{i}^{n+1}$ as function of the lengths $l_{i}^{n}$ and $l_{i}^{n+1}$ respectively. Therefore given three length $l_{i}^{n}$ the momenta $p_{i}^{n}$ are determined by the constraints, whereas the three length $l_{i}^{n+1}$ at the next time step can be chosen freely (respecting generalized triangle inequalities). This corresponds to a free choice of lapse and shift at the vertex $v^{n}$. The set of six length variables $l_{i}^{n}, l_{i}^{n+1}$ determines the length of the tent pole $t^{n}$.

Moreover one can check explicitly that the constraints (6.3) are first class, even Abelian (see appendix (B). See also [31, where it is shown that taking into account constraints based at different vertices we obtain a first class algebra. The constraints are the infinitesimal generators for time evolution - the free choice of the three Lagrange multipliers corresponds to the gauge choice of the three edge lengths $l_{n}^{n+1}$ in the equations (6.2). The choice of only one of the three Lagrange multipliers $N_{j}=\varepsilon$ for the constraints $C_{i}=p_{i}^{n}+\pi-\theta_{v^{n} i}\left(l_{i}^{n}, s_{i j}\right)$ to be non-vanishing corresponds to the choice $l_{k \neq j}^{n+1}=l_{k \neq j}^{n}, l_{j}^{n+1}=l_{j}+\varepsilon$ for the lengths at the next (infinitesimal) time step.

If we switch to a non-vanishing cosmological constant the solutions of the Regge equations do not display the same gauge freedom. This has consequences for the canonical analysis.

The difference to the flat case is that if we solve (numerically) the second equation of (6.2) for the length of the tent pole and use this solution $t^{n}\left(l_{i}^{n}, l_{i}^{n+1}\right)$ in the first equation the dependence of the momenta $p_{i}^{n}$ on the lengths $l_{i}^{n+1}$ will not drop out. Hence we can solve these equations for the lengths $l_{i}^{n+1}$ at the next time step as a function of the lengths $l_{i}^{n}$ and momenta $p_{i}^{n}$ at time step $n$. Using the last equation in (6.2) we can then determine also the momenta $p_{i}^{n+1}$ at the next time step $(n+1)$. That is given some initial data $l_{i}^{0}, p_{i}^{n}$ the evolution is unique for all other time steps (ignoring discrete non-uniqueness and assuming the existence of a solution).

Note however that the dependence of the momenta $p_{i}^{n}\left(l_{j}^{n}, l_{j}^{n+1}\right)$ on $l_{j}^{n+1}$ is very weak (for small $\lambda$ ), see Figure 8. Indeed as we will see later, if we expand $p_{i}^{n}\left(l_{j}^{n}, l_{j}^{n+1}\right)$ in $\lambda$ only the second and higher order terms will depend on $l_{j}^{n+1}$.

Figure 8 shows the functions $p_{i}^{n}\left(l_{j}^{n}, l_{j}^{n+1}\right)$ evaluated on a homogeneous configuration where $l_{j}^{n}=l^{0}$ and $l_{j}^{n+1}=l^{1}$ as well as $s_{i j}=1$. We plotted the momenta as functions of $l^{0}$, but for different values of $l^{1}$ ranging from $l^{0}+0.01$ to $l^{0}+1.2$. This is a wide range considering that $l_{0}$ varies between 0.8 and 2. Moreover we used $\lambda=0.6,1.2$ and $\lambda=2.4$. The four plots at the top in Figure 8 are for the highest value of the cosmological constant $\lambda$ and show quite a variation with $l^{1}$. For $\lambda=1.2$ the variation is much smaller (the family of plots below the four top plots). The plots for $l^{1}=l^{2}+0.01$ and $l^{1}=l^{0}+0.4$ are almost above each other. Choosing canonical data outside of the region traced out by the plots would lead to an evolution with large edge

\footnotetext{
${ }^{3}$ This can be seen from the fact that $S_{(n, n+1)}$ is symmetric under exchange of $l_{i}^{n}$ and $l_{i}^{n+1}$. The different signs in the definition of momenta in (6.2) are absorbed by the property of the momenta to change sign for evolution in 'forward' or 'backward' direction. See also the discussion for the momenta to first order in $\lambda$.
} 


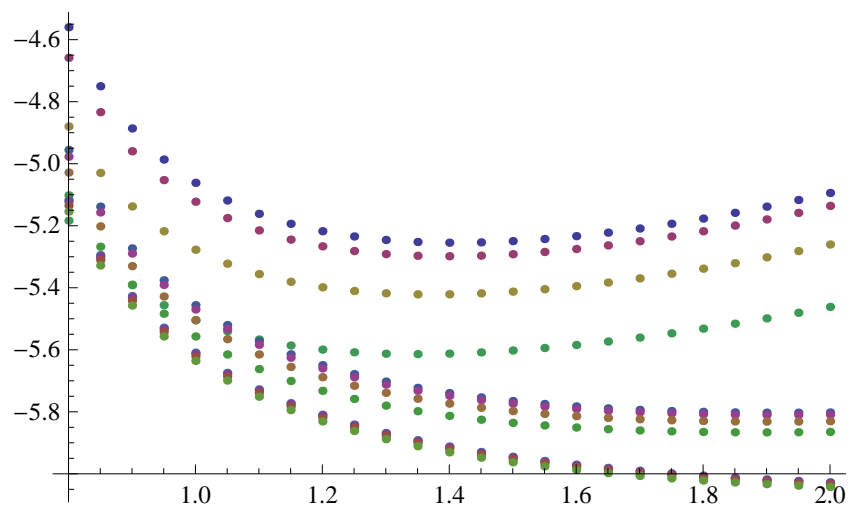

Figure 8: The momentum $p^{0}$ as a function of the length $l^{0}$ for $\lambda=0.6,1.2,2.4$ and $l^{1}=l^{0}+$ $0.01,0.4,0.8,1.2$.

length or would not allow for any (real) solutions. Finally for $\lambda=0.6$ all the four plots are almost above each other.

That is although we can apparently freely choose initial data $l_{i}^{0}, p_{i}^{0}$ a corresponding solution might not exist or lead to very large edge lengths. If one wants for instance the lengths $l_{i}^{1}$ at the following time step to be in rather small intervals around $l_{i}^{0}$ (such that the length $t^{0}$ is small), the momenta $p_{i}^{0}$ will be also quite restricted to a small interval. For the flat case we argued that the gauge choice of the $l_{i}^{n+1}$ corresponds to a choice of the Lagrange multipliers lapse and shift. If we consider a non-vanishing cosmological constant we can interpret the functions

$$
C_{i}^{\lambda}=p_{i}^{n}-p_{i}^{n}\left(l_{j}^{n}, l_{j}^{n+1}\right)
$$

as lapse and shift dependent constraints. For lapse and shift in small intervals we will obtain instead of one constraint hypersurface of infintesimal width a family of hypersurfaces labeled by lapse and shift or in other words a thickened constraint hypersurface of finite width. In the limit of vanishing cosmological constant (but keeping the lapse and shift intervals fixed) the width of this thickened constraint hypersurface converges to zero.

Next we will discuss limits in which one may regain proper constraints. To obtain such constraints for finite $\lambda$ one might try to get rid of the $l_{i}^{n+1}$-dependence of the pseudo constraint $C_{i}^{\lambda}$ in (6.5) by considering the limit $l_{i}^{n+1} \rightarrow l_{i}^{n}$ (in case it is well defined). It corresponds to a limit in which the discretization scale in time direction goes to zero (whereas the discretization scale in spatial direction is fixed). Such a limit does indeed work for discretizations of reparametrization invariant systems and moreover is suggested by the plots in Figure 8 ,

For the system considered here these considerations do not lead to useful results however. A reason for this can be found by considering the behavior of the eigenvalues of the Hessian of the action. That is we consider two consecutive time steps and consider the Hessian associated to the inner vertex. In Figures 9,10 we plot the eigenvalues of this Hessian, that correspond to translation of the inner vertex in time like and space-like directions respectively as a function of the length of the second tent pole. (The cosmological constant is $\lambda=0.5$, and we again consider a homogeneous solution with $l_{i}^{0}=1, s_{i j}=1$.) The eigenvalue for the time-like direction - corresponding to the Hamiltonian constraint - does indeed go to zero with the length of the tent pole. The eigenvalues corresponding to the spatial diffeomorphism constraints however start rather to grow for very small lengths of the tent pole. Hence in this kind of limit only one of the three gauge symmetries seems to get restored 4 This is in agreement with other arguments [11] claiming that for a restoration of diffeomorphism invariance for Regge calculus one needs to

\footnotetext{
${ }^{4}$ Note that if we consider a "symmetry reduction" of the system, in which all the $s_{i j}$ are the same as well as the
} 
consider triangulations in which the simplices are "fat" enough, i.e. are not degenerated. But in the limit we are considering here the tetrahedra become infinitely thin in time-like directions.

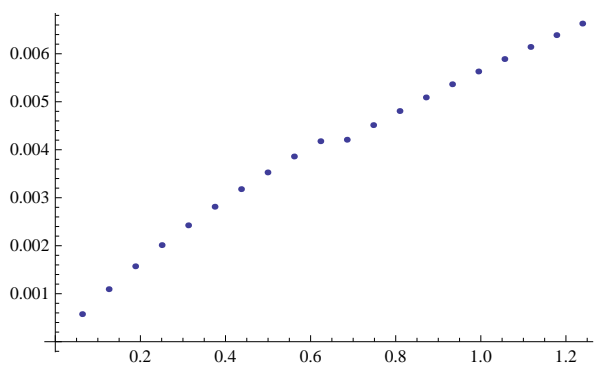

Figure 9: The eigenvalue corresponding to translations in time-like direction as function of the tent pole variable.

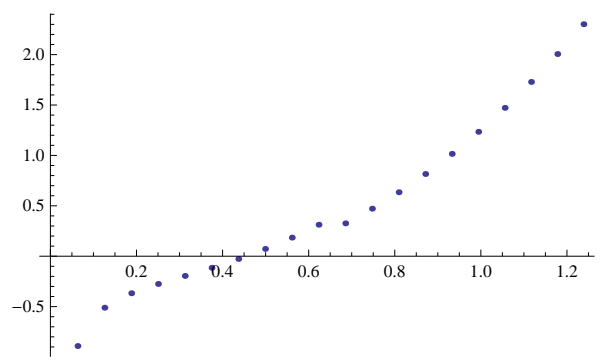

Figure 10: The eigenvalues corresponding to translations in space-like direction as a function of the tent pole variable.

For the dynamics defined by the standard 3d Regge action with a non-vanishing cosmological constant we do not obtain constraints by trying to take the limit of a vanishing discretization scale in time like direction while the spatial discretization scale is kept fixed. (For actions with an exact gauge invariance such a limit can be trivially obtained as there arbitrary values for the lapse functions are allowed and hence also infinitesimal values.) We can also consider the limit in which both the time-like and space-like discretization scale is very small. By rescaling the length variables it is easy to see that this corresponds to taking the cosmological constant $\lambda$ to be small. Hence to consider this limit we can perform an expansion of the constraints in $\lambda$. This expansion also allows us to find the constraints to a certain order explicitly.

The zeroth order of the constraints is given by the flat dynamics (6.3). To find the first order we will make the ansatz $t^{n}={ }^{0} t^{n}+\lambda^{1} t^{n}$ in the second equation of (6.2), expand everything to first order and solve for ${ }^{1} t^{n}$. We use this result

$$
{ }^{1} t^{n}=\left(\frac{\partial \epsilon_{t}^{n}}{\partial t^{n}}\right)^{-1} \frac{\partial V_{(n, n+1)}}{\partial t^{n}} \mid t^{n}={ }^{0} t^{n}
$$

in the first equation of (6.2) and expand again to first order in $\lambda$ :

$$
p_{i}^{n}=-\pi+\theta_{v^{n} i}\left(l_{i}^{n}, s_{i j}\right)+\lambda\left(\frac{\partial \psi_{i}^{n+}}{\partial t^{n}}\left(\frac{\partial \epsilon_{t}^{n}}{\partial t^{n}}\right)^{-1} \frac{\partial V_{(n, n+1)}}{\partial t^{n}}-\frac{\partial V_{(n, n+1)}}{\partial l_{i}^{n}}\right)_{\mid t^{n}=0^{0} t^{n}}+O\left(\lambda^{2}\right) .
$$

Although the explicit expression (6.7) as function of the length variables looks quite lengthy (see appendix B detailing the derivatives of simplex volumes and dihedral angles) the dependence of the first order term on the length variables $l_{j}^{n+1}$ at the upper time step drops out. This can be seen by rewriting the derivative $\frac{\partial \psi_{i}^{n+}}{\partial t^{n}}$ in (6.7) as

$$
\begin{aligned}
\frac{\partial \psi_{i}^{n+}}{\partial t^{n}} & =-\frac{\partial \theta_{v^{n} v^{n+1} i j}^{v^{n}}}{\partial l_{v^{n} v^{n+1}}}-\frac{\partial \theta_{v^{n}}^{v^{n} v^{n+1} i k}}{\partial l_{v^{n} v^{n+1}}} \\
& =-\frac{\partial \theta_{v^{n} v^{n+1}}^{v^{n}} i j}{\partial l_{v^{n} i}}-\frac{\partial \theta_{v^{n} v^{n+1}}^{v^{n}} i k}{\partial l_{v^{n} i}} \\
& =\frac{\partial \epsilon_{t}^{n}}{\partial l_{i}^{n}}
\end{aligned}
$$

$l_{i}^{n}$ we reduce the (potential) gauge freedom to translations in time direction. This is the situation used for the plot in Figure 8 In this case one can indeed find the time continuous limit and hence a time reparametrization invariant system, as the gauge freedom for these translations is restored for infinitesimal time steps. However beginning with the second order in $\lambda$ the resulting Hamiltonian does not coincide with the corresponding Hamiltonian constraint obtained from a symmetry reduction of the alternative dynamic with exact gauge invariance, see below. 
where we used that $\frac{\partial \theta_{e}^{\tau}}{\partial l_{e^{\prime}}}=\frac{\partial \theta_{e^{\prime}}^{\tau}}{\partial l_{e}}$ for the dihedral angles $\theta_{e}^{\tau}, \theta_{e^{\prime}}^{\tau}$ at the edges $e, e^{\prime}$ respectively of a tetrahedron $\tau$ (see appendix (B). In (6.8) the tetrahedron $\tau$ is the one with vertices $v^{n} v^{n+1} i j$ or $v^{n} v^{n+1} i k$ and $e$ the edge between vertices $v^{n} i$ and $e^{\prime}$ between $v^{n} v^{n+1}$.

Now if we denote by ${ }^{0} t$ the function of the variables $l_{j}^{n}, l_{j}^{n+1}$ that results by solving the equation $\epsilon_{t}^{n}\left(t^{n}, l_{j}^{n}, l_{j}^{n+1}\right)=0$ for $t^{n}$, we find by taking the total derivative of this equation with respect to $l_{i}^{n}$ that

$$
\frac{\partial^{0} t}{\partial l_{i}^{n}}=-\frac{\partial \epsilon_{t}^{n}}{\partial l_{i}^{n}}\left(\frac{\partial \epsilon_{t}^{n}}{\partial t^{n}}\right)_{\mid t^{n}={ }^{0} t^{n}}^{-1}
$$

Using (6.8) and (6.8) we have for the first order term in (6.7)

$$
\begin{aligned}
\left(\frac{\partial \psi_{i}^{n+}}{\partial t^{n}}\left(\frac{\partial \epsilon_{t}^{n}}{\partial t^{n}}\right)^{-1} \frac{\partial V_{(n, n+1)}}{\partial t^{n}}-\frac{\partial V_{(n, n+1)}}{\partial l_{i}^{n}}\right)_{\mid t^{n}={ }^{0} t^{n}} & =\left(-\frac{\partial V_{(n, n+1)}}{\partial t^{n}} \frac{\partial^{0} t}{\partial l_{i}^{n}}-\frac{\partial V_{(n, n+1)}}{\partial l_{i}^{n}}\right)_{\mid t^{n}={ }^{0} t^{n}} \\
& =-\frac{\mathbf{d} V_{(n, n+1)}\left({ }^{0} t\left(l_{j}^{n}, l_{j}^{n+1}\right), l_{j}^{n}, l_{j}^{n+1}\right)}{\mathbf{d} l_{i}^{n}} .
\end{aligned}
$$

The volume $V_{(n, n+1)}\left({ }^{0} t\left(l_{j}^{n}, l_{j}^{n+1}\right), l_{j}^{n}, l_{j}^{n+1}\right)$ is the one of a flat "double pyramid", depicted in Figure 7. For the kind of orientation of the two tetrahedra as shown in this figure this volume is just the difference of the volumes of the two tetrahedra with length $l_{j}^{n}, s_{i j}$ and $l_{j}^{n+1}, s_{i j}$ respectively. Therefore the final expression for the momenta to first order in $\lambda$ is then

$$
p_{i}^{n}=-\pi+\theta_{v^{n} i}\left(l_{j}^{n}, s_{i j}\right)+\lambda \frac{\partial V\left(l_{j}^{n}, s_{i j}\right)}{\partial l_{i}^{n}}+O\left(\lambda^{2}\right)
$$

where $V\left(l_{j}^{n}, s_{i j}\right)$ is the volume of a tetrahedron $\tau$ with edge lengths $\left(l_{j}^{n}, s_{i j}\right)$. Note that the constraints truncated to first order are also first class and even Abelian 5 This can be easily seen by realizing that $S_{\tau}=\sum_{e \in \tau} l_{e}\left(\pi-\theta_{e}^{\tau}\right)-\lambda V^{\tau}$ is a generating function for the first order momenta, that is $p_{i}^{n}=-\partial S_{\tau} / \partial l_{i}^{n}+O\left(\lambda^{2}\right)$.

Furthermore the constraints are preserved under time evolution. Note that the action $S_{(n, n+1)}$ is symmetric under the exchange of the variables $l_{i}^{n}$ and $l_{i}^{n+1}$. For the first order of the momenta $p_{i}^{n+1}$ as defined in the third equation of (6.2) we therefore obtain

$$
{ }^{1} p_{i}^{n+1}=\frac{\mathbf{d} V_{(n, n+1)}\left({ }^{0} t\left(l_{j}^{n}, l_{j}^{n+1}\right), l_{j}^{n}, l_{j}^{n+1}\right)}{\mathbf{d} l_{i}^{n+1}}
$$

that is again the derivative of the volume $\left.V_{(n, n+1}\right)=V\left(l_{j}^{n+1}, s_{i j}\right)-V\left(l_{j}^{n}, s_{i j}\right)$ of a flat double pyramid (but this time with a plus sign). We have however to take the derivative with respect to the length $l_{i}^{n+1}$, that affects the larger tetrahedron of this double pyramid. In the end we obtain the same sign as in (6.11)

$$
p_{i}^{n+1}=-\pi+\theta_{v^{n+1} i}\left(l_{j}^{n+1}, s_{i j}\right)+\lambda \frac{\partial V\left(l_{j}^{n+1}, s_{i j}\right)}{\partial l_{i}^{n+1}}+O\left(\lambda^{2}\right) .
$$

The constraints (6.12) coincide to first order with a first order expansion in $\lambda$ of the constraints describing an exact discretization of $3 \mathrm{~d}$ gravity with cosmological constant [30]. The

\footnotetext{
${ }^{5}$ Note that we did not check the commutator between constraints based at neighbouring vertices. If the 'Cauchy surface' we are considering is the surface of a tetrahedron, we would get the same first order constraints for the four three-valent vertices and these would still be Abelian.
} 
continuum solutions of this theory are spaces with homogeneous and constant curvature determined by the cosmological constant. Accordingly instead of a flat tetrahedron embedded in flat space, we consider a tetrahedron with homogeneously curved geometry embedded in a space with the same kind of geometry. In analogy with the constraints (6.4) for the flat geometry, which fix the momenta to agree with the dihedral angles of a flat tetrahedron, the constraints in this case fix the momenta to agree with the dihedral angles of a homogeneously curved tetrahedron

$$
{ }^{e x a c t} p_{i}^{n}=-\pi+{ }^{\lambda} \theta_{v^{n} i}\left(l_{j}^{n}, s_{i j}\right)
$$

(see appendix B for expressions giving the dihedral angles ${ }^{\lambda} \theta_{e}$ for a tetrahedron in homogeneously curved space as a function of the edge lengths). An expansion of these constraints to first order in the curvature or cosmological constant gives (6.11). To see this one needs the identity

$$
\frac{\mathbf{d}^{\lambda} \theta_{e}^{\tau}}{\mathbf{d} \lambda}=\frac{\partial^{\lambda} V}{\partial l_{e}}
$$

where ${ }^{\lambda} V$ is the volume of a tetrahedron in homogeneously curved space, that will be derived in appendix B.

Starting with second order in $\lambda$ the (pseudo) constraints (6.5) do depend on the length variables $l_{i}^{n+1}$ at the upper time step. (This can be more easily checked by considering the "symmetry reduced" theory, where all $s_{i j}=s$ and all $l_{i}^{n}=l^{n}$, see also [20].)

To summarize, we have seen that the canonical equations of motions for the tent moves reflects the gauge symmetries of the covariant theory. If the symmetries are exact, we will encounter proper first class constraints. (See also next section for a general derivation.) These constraints generate translations of the evolved vertex and hence mirror the non-uniqueness of the covariant solutions. If the gauge symmetries are broken, we do not obtain constraints in the usual sense, as the expressions depend on the lenght variables on the next time step, or equivalently on lapse and shift. This again mirrors that the covariant solutions are unique (ignoring discrete cases of non-uniqueness) and hence lapse and shift are fixed by the pseudo constraints. However to obtain reasonable (small) values for lapse and shift, the initial data have to be chosen carefully - effectively from a "thickened" constraint hypersurface of finite width.

Trying to construct a time continuum limit in order to obtain proper constraints however fails in the case of $3 \mathrm{~d}$ Regge calculus with cosmological constant. The reason is that not all gauge symmetries are restored in this limit, in which the time discretization scale goes to zero whereas the spatial discretization scale is fixed.

If we take all edge length to be small, or equivalently consider a small cosmological constant, we can perform a perturbation in the cosmological constant $\lambda$. The dynamics truncated to first order has gauge symmetries and we can obtain constraints. For 3d Regge calculus with cosmological constant exact constraints (reflecting exactly the continuum dynamics) exist [30] and are a higher order continuation of the first order constraints derived here.

We will show in 28] that a similar expansion in curvature is also possible for $4 \mathrm{~d}$ Regge calculus. In this case we do not know the exact (discretized) constraints but if we are able to construct the first order constraints the question arises if higher order terms can be derived and whether these are determined uniquely. These questions will be subjects for further research.

\section{Relation between symmetries of the action and constraints in the canonical framework}

Here we will discuss the relation between gauge symmetries of the action and constraints in more detail. 
We argued that gauge symmetries should lead to a non-trivial action of the gauge group on solutions. As we are considering continuous groups this should result in non-unique solutions parametrized by the gauge group parameters. Since solutions are equivalent with extrema of the action, rather than having one isolated extremum there is a submanifold of extrema on which the action is constant. As the first derivatives of the action vanishes by definition we obtain as a necessary condition for continuous gauge symmetries that the Hessian of the action should have null directions.

We will consider a triangulation obtained from two consecutive tent moves. Hence we will have edges with length $l_{e}^{n-1}$ and $l_{e}^{n+1}$ in the 'lower' and 'upper' boundary of the triangulation respectively. Here $e$ is an index for the edges from the evolved vertex $v$ to the adjacent vertices. At the inner vertex $v^{n}$ will hinge edges with the length $l_{e}^{n}, t^{n-1}$ and $t^{n}$. Denoting with $S=$ $S_{(n-1, n)}+S_{(n, n+1)}$ the action with boundary terms for this triangulation the requirement for a null vector $Y^{\alpha}, \alpha=e^{n}, t^{n-1}, t^{n}$ gives the equations

$$
\begin{aligned}
& \sum_{e} Y^{e} \frac{\partial^{2} S}{\partial l_{e}^{n} \partial l_{e^{\prime}}^{n}}+Y^{t^{n-1}} \frac{\partial^{2} S}{\partial t^{n-1} \partial l^{\prime}}+Y^{t^{n}} \frac{\partial^{2} S}{\partial t^{n} \partial l^{e^{\prime}}}=0 \\
& \sum_{e} Y^{e} \frac{\partial^{2} S}{\partial l_{e}^{n} \partial t^{n-1}}+Y^{t^{n-1}} \frac{\partial^{2} S}{\partial t^{n-1} \partial t^{n-1}}=0 \\
& \sum_{e} Y^{e} \frac{\partial^{2} S}{\partial l_{e}^{n} \partial t^{n}}+Y^{t^{n}} \frac{\partial^{2} S}{\partial t^{n} \partial t^{n}}=0
\end{aligned} .
$$

The momenta conjugated to the tent pole variables have to vanish and the length of the tent poles do not appear as boundary data. Hence these variables are not fully dynamical. Therefore we will first integrate out these variables.

We solve for the lengths of the tent poles $t^{n-1}$ and $t^{n}$ the equations of motions

$$
\begin{aligned}
& \frac{\partial S_{(n-1, n)}}{\partial t^{n-1}}=0 \\
& \frac{\partial S_{(n, n+1)}}{\partial t^{n}}=0 .
\end{aligned}
$$

Using the solutions $T^{n-1}\left(l_{e}^{n-1}, l^{n}\right)$ and $T^{n}\left(l_{e}^{n}, l_{e}^{n+1}\right)$ in (7.2) and differentiating these identities with respect to $l_{e}^{n}$ we obtain

$$
\begin{aligned}
& \frac{\partial^{2} S_{(n-1, n)}}{\partial t^{n-1} \partial l_{e}^{n}}+\frac{\partial^{2} S_{(n-1, n)}}{\partial t^{n-1} \partial t^{n-1}} \frac{\partial T^{n-1}}{\partial l_{e}^{n}}=0 \\
& \frac{\partial^{2} S_{(n, n+1)}}{\partial t^{n} \partial l_{e}^{n}}+\frac{\partial^{2} S_{(n, n+1)}}{\partial t^{n} \partial t^{n}} \frac{\partial T^{n}}{\partial l_{e}^{n}}=0 .
\end{aligned}
$$

The resulting effective action is

$$
\tilde{S}\left(l_{e}^{n-1}, l_{e}^{n}, l_{e}^{n+1}\right):=S\left(l_{e}^{n-1}, l_{e}^{n}, l_{e}^{n+1}, T^{n-1}\left(l_{e}^{n-1}, l_{e}^{n}\right), T^{n}\left(l_{e}^{n}, l_{e}^{n+1}\right)\right) .
$$

Using the equations (7.3) the Hessian $\tilde{H}$ of the modified action $\tilde{S}$ can be written as

$$
\frac{\partial^{2} \tilde{S}}{\partial l_{e}^{n} \partial l_{e^{\prime}}^{n}}=\frac{\partial^{2} S}{\partial l_{e}^{n} \partial l_{e^{\prime}}^{n}}+\frac{\partial^{2} S}{\partial l_{e}^{n} \partial t^{n-1}} \frac{\partial T^{n-1}}{\partial l_{e^{\prime}}^{n}}+\frac{\partial^{2} S}{\partial l_{e}^{n} \partial t^{n}} \frac{\partial T^{n}}{\partial l_{e^{\prime}}^{n}} \text {. }
$$

Finally with the help of the three equations in (7.1) and again (7.3) it follows that null vectors for the Hessian $H$ of $S$ define also null vectors for the Hessian $\tilde{H}$ of $\tilde{S}$ :

$$
\sum_{e} Y^{e} \frac{\partial^{2} \tilde{S}}{\partial l_{e}^{n} \partial l_{e^{\prime}}^{n}}=0
$$


Because of the second and third equations in (7.1) we can express the components $Y^{t^{n-1}}$ and $Y^{t^{n}}$ as a combination of the components $Y^{e}$. (Here we assume that the second partial derivatives of the action with respect to $t^{n-1}$ and $t^{n}$ do not vanish. This is generically the case for the Regge action.) For this reason a set of linearly independent null vectors for the Hessian $H$ will define a set of independent null vectors for the Hessian $\tilde{H}$ of the same size.

We will use the action $\tilde{S}$ to find stationary points

$$
L_{e}^{n}\left(l_{e}^{n-1}, l_{e}^{n+1}\right)
$$

as functions of the boundary data $l_{e}^{n-1}, l_{e}^{n+1}$. Again we use these solutions in the equations of motion

$$
\frac{\partial \tilde{S}}{\partial l_{e}^{n}}\left(l_{e}^{n-1}, L_{e}^{n}\left(l_{e}^{n-1}, l_{e}^{n+1}\right) l^{n+1}\right)=0
$$

and differentiate these identities with respect to $l_{e^{\prime \prime}}^{n+1}$ :

$$
\sum_{e^{\prime}} \frac{\partial^{2} \tilde{S}}{\partial l_{e}^{n} \partial l_{e^{\prime}}^{n}} \frac{\partial L_{e^{\prime}}^{n}}{\partial l_{e^{\prime \prime}}^{n+1}}+\frac{\partial^{2} \tilde{S}_{(n, n+1)}}{\partial l_{e}^{n} \partial l_{e^{\prime \prime}}^{n+1}}=0
$$

That is we have

$$
\tilde{H} \cdot L=-K
$$

where $L_{e e^{\prime}}:=\partial L_{e}^{n} / \partial l_{e^{\prime}}^{n+1}$ and

$$
K_{e e^{\prime}}:=\frac{\partial^{2} \tilde{S}_{(n, n+1)}}{\partial l_{e}^{n} \partial l_{e^{\prime}}^{n+1}} .
$$

Therefore null eigenvectors for the Hessian $\tilde{H}$ are left null eigenvectors for the matrix $K$.

Note that these considerations show that $K$ has at least as many null vectors as the Hessian $H$. It is not excluded that $K$ has even more null vectors, however that was not the case in the examples we investigated.

The matrix $K$ appears as the matrix of derivatives of the momenta

$$
p_{e}^{n}:=-\frac{\partial \tilde{S}_{(n, n+1)}}{\partial l_{e}^{n}}
$$

with respect to the length variables $l_{e^{\prime}}^{n+1}$. If there exist null vectors we cannot expect to be able to solve the equations (7.12) for the lengths $l_{e}^{n+1}$ at the next time step as a function of the momenta and lengths at time $n$ as the conditions of the implicit function theorem are violated.

If we assume that the rank of the matrix $K$ is constant (and not maximal) in a neighbourhood of the initial data set $\left(l_{e}^{n}, l_{e}^{n+1}\right)$ under consideration then we can conclude that the Legendre transform from the direct product of configuration spaces $Q^{n} \times Q^{n+1}$ to the cotangent space $T^{*}\left(Q^{n}\right)$ defined by

$$
\left(l_{e}^{n}, l_{e}^{n+1}\right) \mapsto\left(l_{e}^{n}, p_{e}^{n}\right)
$$

is neither injective nor surjective.

The image of this map into the phase space is the (primary) constraint hypersurface. The number of (irreducible) constraints describing this surface is larger or equal to the number of linearly independent null vectors for the Hessian of the action in (7.1). The constraints are relations between the momenta and length variables at the same time step $n$ and will follow from the equations (7.12).

This shows that if the action has (local) gauge symmetries, we will obtain proper constraints on the canonical data. (For general actions there might also appear constraints without having 
gauge symmetries.) Also we cannot solve the relation (7.12) uniquely for the length $l_{e}^{n+1}$ as a function of the data $l_{e}^{n}, p_{e}^{n}$ at time step $n$. Hence there will appear arbitrary parameters (Lagrange multipliers) $\lambda^{\alpha}$ in the relations expressing $l^{n+1}$ as a function of $l_{e}^{n}, p_{e}^{n}$. Whether all these Lagrange multipliers remain arbitrary or not depends on how many of the constraints are preserved by time evolution. So we have the same number of constraints as before following from the relations

$$
p_{e}^{n+1}=\frac{\partial \tilde{S}_{(n, n+1)}}{\partial l_{e}^{n+1}}
$$

If these constraints coincide with the ones derived from (17.12) then we obtain no further constraints and the Lagrange multipliers remain arbitrary, as the constraints are automatically preserved by time evolution. In general it might however happen that new (secondary) constraints appear or some of the Lagrange multipliers get fixed, see also [18] for an extensive discussion. For the case considered here - as the canonical dynamics is just a reformulation of the covariant Regge equations - we should obtain the same number of free Lagrange parameters as null vectors for the Hessian. This was indeed the case for the examples considered in section 6.

\section{Discrete reparametrization invariant systems}

We so far discussed examples from Regge calculus where for curved solutions we did not find an exact form of gauge invariance. One could argue that the reason for this is that in Regge calculus one already operates on the space of diffeomorphism invariant geometries, hence one would not expect any gauge symmetries to appear.

If we adopt this view it might be however very difficult to understand the continuum limit. In particular for topological theories, such as $3 \mathrm{~d}$ gravity with cosmological constant it is hard to see how in a refinement limit that introduces more and more degrees of freedom (that would all be physical) one can obtain a continuum theory, in which only a finite and very limited number of degrees of freedom are actually physical.

Indeed one expects $[10,11,12$ that in a continuum limit the (continuum) gauge symmetries are restored. The arguments are based on the fact that in a refinement limit the "curvature per simplex", that is the deficit angles are getting very small (with the edge lengths getting small as compared to the average curvature scale). We have seen that the eigenvalues of the Hessian, that correspond to the broken or 'would-be' symmetries go to zero (quadratically) with the deficit angles.

The viewpoint that discretizations lead to a breaking of gauge symmetries that are then restored in the continuum limit is also strengthened by considering discretizations of parametrized (finite dimensional) systems. In those cases the reparametrization invariance is generically broken for the discretized systems. One could also argue that one is somehow working on the space of gauge invariant data, there is however always a choice of action for which reparametrization invariance is fully restored! This shows that it is important to keep in mind that the question of gauge symmetry or gauge invariance is one determined by the dynamics - that is the action - of the system.

We will give a short discussion of discretized reparametrization invariant systems, more material and applications to numerical integration can be found in [25].

To obtain a continuous action with reparametrization invariance we can start from a regular Lagrangian $L(q, \dot{q})$ where $q$ denote the configuration variables. To obtain a reparametrization invariant action we add the time variable $t$ to the configuration variables and use $s$ as an 
(auxiliary) evolution parameter instead. It is then straightforward to verify, that

$$
S=\int_{s_{i}}^{s_{f}} L\left(q, \frac{q^{\prime}}{t^{\prime}}\right) t^{\prime} d s
$$

is indeed invariant under reparametrizations $\tilde{s}=f(s)$ of the evolution parameter and the induced change $\tilde{t}(\tilde{s})=t\left(f^{-1}(\tilde{s})\right), \tilde{q}(\tilde{s})=q\left(f^{-1}(\tilde{s})\right)$ of the evolution pathes. Here a prime denotes differentiation with respect to $s$.

One family of discretizations of this action is given by

$$
S_{d}=\sum_{n=0}^{N-1} S_{(n, n+1)}
$$

with

$$
S_{(n, n+1)}:=\left(t^{n+1}-t^{n}\right) L\left((1-\alpha) q^{n}+\alpha q^{n+1}, \frac{q^{n+1}-q^{n}}{t^{n+1}-t^{n}}\right) .
$$

The (Euler-Lagrange) equations of motion are then given by second order difference equations which can be obtained from

$$
\begin{aligned}
0 & =\frac{\partial S_{(n-1, n)}}{\partial t^{n}}+\frac{\partial S_{(n, n+1)}}{\partial t^{n}} \\
0 & =\frac{\partial S_{(n-1, n)}}{\partial q^{n}}+\frac{\partial S_{(n, n+1)}}{\partial q^{n}}
\end{aligned} .
$$

As an example consider the discretized reparametrization invariant harmonic oscillator with a discrete action for one time step given by

$$
S_{(n, n+1)}=\left(\frac{1}{2} \frac{\left(q^{n+1}-q^{n}\right)^{2}}{\left(t^{n+1}-t^{n}\right)^{2}}-\frac{1}{2} w\left(\frac{1}{2} q^{n}+\frac{1}{2} q^{n+1}\right)^{2}\right)\left(t^{n+1}-t^{n}\right)
$$

where $w$ parametrizes the strength of the potential term.

Assume we consider the smallest boundary value problem with prescribed data at time steps $(n-1)$ and $(n+1)$. A necessary condition for the system to be reparametrization invariant is that the Hessian of the action with respect to the variables at the time step $n$ has a null vector if evaluated on solutions. Now basically the same discussion as in section 7 will show that if this Hessian has a null vector, this is also the case for the matrix

$$
K:=\left(\begin{array}{cc}
\frac{\partial^{2} S_{(n, n+1)}}{\partial q^{n} \partial q^{n+1}} & \frac{\partial^{2} S_{(n+1)}}{\partial q^{n} \partial t^{n+1}} \\
\frac{\partial^{2} S_{(n, n+1)}}{\partial t^{n} \partial q^{n+1}} & \frac{\partial^{2} S_{(n, n+1)}}{\partial t^{n} \partial t^{n+1}}
\end{array}\right) .
$$

(To consider this matrix has the advantage that we do not need to determine the solution.) An explicit calculation gives for the determinant of $K$

$$
\operatorname{det}(K)=\frac{1}{4} w \frac{\left(q^{n+1}-q^{n}\right)^{2}}{\left(t^{n+1}-t^{n}\right)^{2}}+\frac{1}{4} w^{2}\left(\frac{1}{2} q^{n+1}+\frac{1}{2} q^{n}\right),
$$

that is a non-vanishing result. Hence the discrete system does not display (local) gauge symmetries. Note that for a free particle, $w=0$, reparametrization invariance persists for the discretized theory. As we will see later on the reason is that the dynamic for a free particle defined by the discretized theory coincides with the dynamic of the continuum theory.

This raises the question whether for instance similarly to the case of $3 \mathrm{~d}$ gravity with cosmological constant discussed in section 6, the gauge symmetry and therefore constraints persist for a first order truncation in $w$. It will turn out that this is not the case. On the other hand 
one can define the time continuum limit without any problems and recover reparametrization invariance.

To define time evolution in a canonical formalism we again use the discrete action for one time slice as a generating function:

$$
\begin{aligned}
p_{q}^{n} & =-\frac{\partial S_{(n, n+1)}}{\partial q^{n}}=\frac{\left(q^{n+1}-q^{n}\right)}{\left(t^{n+1}-t^{n}\right)}+\frac{1}{2} w\left(\frac{1}{2} q^{n+1}+\frac{1}{2} q^{n}\right)\left(t^{n+1}-t^{n}\right) \\
p_{t}^{n} & =-\frac{\partial S_{(n, n+1)}}{\partial t^{n}}=-\frac{1}{2} \frac{\left(q^{n+1}-q^{n}\right)^{2}}{\left(t^{n+1}-t^{n}\right)^{2}}-\frac{1}{2} w\left(\frac{1}{2} q^{n+1}+\frac{1}{2} q^{n}\right)^{2} \\
p_{q}^{n+1} & =\frac{\partial S_{(n, n+1)}}{\partial q^{n+1}}=\frac{\left(q^{n+1}-q^{n}\right)}{\left(t^{n+1}-t^{n}\right)}-\frac{1}{2} w\left(\frac{1}{2} q^{n+1}+\frac{1}{2} q^{n}\right)\left(t^{n+1}-t^{n}\right) \\
p_{t}^{n+1} & =\frac{\partial S_{(n, n+1)}}{\partial t^{n+1}}=-\frac{1}{2} \frac{\left(q^{n+1}-q^{n}\right)^{2}}{\left(t^{n+1}-t^{n}\right)^{2}}-\frac{1}{2} w\left(\frac{1}{2} q^{n+1}+\frac{1}{2} q^{n}\right)^{2} .
\end{aligned}
$$

For the free particle $w=0$ the first two equations in (8.8) cannot be solved uniquely for $\left(q^{n+1}, t^{n+1}\right)$ and one is left with a one-parameter ambiguity. Accordingly there is one constraint

$$
C_{w=0}=p_{t}^{n}+\frac{1}{2}\left(p_{q}^{n}\right)^{2}=0
$$

between the canonical data at any time step $n$. For $w>0$ there are at most discrete ambiguities in the solutions $\left(q^{n+1}, t^{n+1}\right)$ as functions of the canonical data at time step $n$. To check whether we obtain a constraint in a first order truncation in $w$ we expand

$$
q^{n+1}={ }^{0} q^{n+1}+w^{1} q^{n+1} \quad, \quad t^{n+1}={ }^{0} t^{n+1}+w^{1} t^{n+1}
$$

where ${ }^{0} t^{n+1}=t^{n}+\left({ }^{0} p_{q}^{n}\right)^{-1}\left({ }^{0} q^{n+1}-q^{n}\right)$ is the solution for the $w=0$ dynamics. We use these expansions in the defintion of the momenta $p_{q}^{n}, p_{t}^{n}$ in (8.8) and neglect any higher than first order terms in $w$. From the resulting equations

$$
\begin{aligned}
& p_{q}^{n}={ }^{0} p_{q}^{n}-w \frac{\left({ }^{1} q^{n+1}-q^{n}\right)^{2}\left({ }^{1} q^{n+1}+q^{n}\right)+4\left({ }^{0} p_{q}^{n}\right){ }^{2}\left({ }^{0} p_{q}^{n}{ }^{1} t^{n}-{ }^{1} q^{n}\right)}{4{ }^{0} p_{q}^{n}\left({ }^{1} q^{n+1}-q^{n}\right)} \\
& p_{t}^{n}=-\frac{1}{2}\left({ }^{0} p_{q}^{n}\right)^{2}+w \frac{\left({ }^{1} q^{n+1}-q^{n}\right)\left({ }^{1} q^{n+1}+q^{n}\right)^{2}+8\left({ }^{0} p_{q}^{n}\right)^{2}\left({ }^{0} p_{q}^{n}{ }^{1} t^{n}-{ }^{1} q^{n}\right)}{8\left({ }^{1} q^{n+1}-q^{n}\right)}
\end{aligned}
$$

we do not obtain a constraint. Rather these can be solved for instance for the variables ${ }^{1} q^{n+1}$ and ${ }^{0} q^{n+1}$ (with ${ }^{1} t^{n+1}$ remaining undetermined). That is these (first order) equations fix the values for the zeroth order variables ${ }^{0} q^{n+1},{ }^{0} t^{n+1}$ which were only determined up to a one-parameter reparametrization invariance by the zeroth order equations. This behaviour differs from the first order dynamics in the cosmological constant for 3d Regge calculus found in section 6 .

On the other hand it is possible to find a continuous time limit, in which reparametrization invariance is restored. (This was not possible for $3 \mathrm{~d}$ Regge calculus with cosmological constant.) Again expanding

$$
q^{n+1}=q^{n}+\varepsilon^{1} q^{n+1} \quad, \quad t^{n+1}=t^{n}+\varepsilon^{1} t^{n+1}
$$

we will find that up to second order terms the relation

$$
C_{\varepsilon}=p_{t}^{n}+\frac{1}{2}\left(p_{q}^{n}\right)^{2}+\frac{1}{2} w\left(q^{n}\right)^{2}+O\left(\varepsilon^{2}\right)=0
$$

between the canonical data. Indeed (8.13) coincides with the continuum constraint for the parametrized harmonic oscillator. 
Finally we want to point out that for such mechanical systems, in which we have only a one-dimensional reparametrization invariance in time direction, it is straightforward to define a discrete dynamics which preserves reparametrization invariance. The idea is that the discrete system should exactly reproduce the dynamics of the continuous system. To achieve such a dynamics one can start with the continuum action and solve for all variables $t(s), q(s)$ except for those at some discrete subsets of the evolution parameter $s_{n}$. Reinserting the solutions in the action will result in a sum of Hamilton-Jacobi functions

$$
S_{\text {exact }}=\sum_{n=0}^{N-1} S_{H J}^{\left(s^{n}, s^{n+1}\right)}\left(t^{n}, q^{n}, t^{n+1}, q^{n+1}\right)=\sum_{n=0}^{N-1} \int_{s_{n}}^{s_{n+1}} d s \mathcal{L}(t(s), q(s)) .
$$

where $\mathcal{L}$ is the Lagrangian of the reparametrization invariant continuous system, and $t(s), q(s)$ are solutions with boundary data $\left(t^{n}, q^{n}, t^{n+1}, q^{n+1}\right)$.

If we define the momenta for this discretized dynamics according to (8.8) we actually obtain the same definition as for the continuums dynamics

$$
\begin{aligned}
& p_{q}^{n}=-\frac{\partial S_{H J}^{\left(s^{n}, s^{n+1}\right)}\left(t^{n}, q^{n}, t^{n+1}, q^{n+1}\right)}{\partial q^{n}} \\
& p_{t}^{n}=-\frac{\partial S_{H J}^{\left(s^{n}, s^{n+1}\right)}\left(t^{n}, q^{n}, t^{n+1}, q^{n+1}\right)}{\partial t^{n}} .
\end{aligned}
$$

Hence the same constraints between the momenta and configuration variables as for the continuum dynamics have to hold, which also shows that reparametrization invariance is preserved.

\section{Discussion}

We have shown that we do not have exact gauge symmetries for non-flat vertices in discretized $4 \mathrm{~d}$ gravity as defined by the Regge action. In a canonical formulation for Regge calculus this will lead to pseudo constraint 6 involving data at different time steps instead of proper constraints involving data at the same time step.

This fact should be considered in attempts to connect covariant and canonical quantum gravity models, for instance loop quantum gravity, based on proper constraints, and spin foam models, that can be seen as quantizations of Regge calculus, which rather lead to pseudo constraints. Indeed the methods discussed in this paper allow to introduce a canonical formalism that completely matches the dynamics of the covariant system and moreover using tent moves makes a local analysis possible. These techniques seem therefore be ideally suited to be applied to other discretized actions, as the discretized Plebanski action [32] used in spin foam models, or the area-angle action for Regge calculus introduced in 33 . This could help to derive a closer connection between spin foam models and loop quantum gravity.

In the case that one starts with an action with broken symmetries and obtains pseudo constraints, there might nevertheless exist certain limiting cases in which these turn into proper constraints. This was the case for the first order dynamics in the cosmological constant for $3 \mathrm{~d}$ Regge calculus. An analysis for 4d Regge calculus for such limiting cases will appear in [28]. Starting from these proper constraints it might be possible to extend the constraints, such that in the end one obtains a system with an alternative dynamics with exact gauge symmetries.

The example of reparametrization invariant systems shows that the breaking of the symmetries in discrete theories is due to deviations of the discrete dynamics from the continuum one.

\footnotetext{
${ }^{6}$ Note that for instance for four-valent tent moves or Cauchy surfaces formed by the boundary of a 4-simplex one will obtain proper constraints restricting the physical degrees of freedom to zero, as the dynamics supported by these structures is just given by $4 \mathrm{~d}$ flat space, see [14].
} 
We discussed examples, where different discretizations of the action lead to a symmetry breaking and symmetry preserving dynamics. Hence one should be careful in deriving general conclusions for quantum gravity models from the fact that the symmetries are broken for the Regge action. Alternative actions might display exact gauge invariance, see also [34] for attempts to obtain such actions.

For deparametrization invariant systems we have seen that such an alternative action can be always defined by starting from the continuum action and integrating out infinitely many degrees of freedom, that is in other words by applying a renormalization group transformation. We will explore this idea in more detail in [30. Indeed in lattice field theories the so-called perfect actions, which reflect exactly the continuum dynamics, can be constructed as fixed points of the renormalization group flow [35]. These lead typically to non-local couplings (i.e. couplings extending over non-adjacent lattice sites). It would be therefore interesting to extend the analysis of this paper to non-local actions.

Note that another property for the exact discretized action (8.14) is the independence under refinements (for the partition function or dynamical observables). Indeed as the dynamics already reflects the continuum dynamics, there is nothing gained by refining the lattice. Similarly one would expect that a perfect action for discretized gravity - should it exist - would lead to triangulation independent partition functions. This is well known for topological field theories (such as 3d gravity), where however the actions are still local. For theories with propagating degrees of freedom non-local couplings might arise (even at the classical level).

An alternative way to obtain triangulation independence for instance for spin foam models, is to sum over triangulations, which would also include infinitely refined triangulations. This can however be only defined at a formal level, for instance by invoking group field theory methods [36. In some sense the construction of a perfect action can be seen as taking the effects of a sum over triangulation into account and might in this way circumvent divergencies that appear in a sum over triangulations. An investigation into the properties of such actions seems therefore promising to us.

\section{A An example for physically different solutions with the same boundary conditions}

In this section we will construct an example of two physically different Regge solutions for the same boundary data. The construction uses two tent moves. In the end we will obtain two solutions, one flat and one with curvature. As the final triangulation has an inner vertex we will check the Hessian of the action evaluated on these solutions and find null eigenvalues for the flat solution and only non-vanishing eigenvalues for the curved solution.

\section{A.1 One tent move}

We consider the following four-dimensional triangulation: start with a regular tetrahedron where all edges have unit length. Apply a 1-4 move, i.e. add another vertex $A$, which is connected to each of the four former vertices $V_{1}, \ldots, V_{4}$. The length of each of the edges from $A$ to $V_{i}$ is $a>0$. This three-dimensional triangulation serves as a starting point for a tent move applied at $A$. The resulting four-dimensional triangulation has yet another vertex $B$, which is connected with each of the $V_{i}$, the length of each of the connecting edges being $b$. Denote the length of the tent pole, i.e. the edge between $A$ and $B$ by $t$.

The tent pole is the only inner edge of this triangulation, and its Regge equation reads 


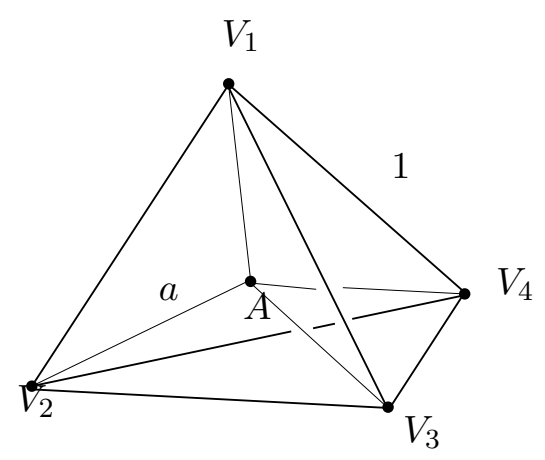

Figure 11: The initial triangulation. The lengths of the edges connecting any $V_{i}$ to any other $V_{j}$ is 1 , the length of the edges connecting $A$ to any of the $V_{i}$ is $a$.

$$
\sum_{i=1}^{4} \frac{\partial A_{A B V_{i}}}{\partial t} \epsilon_{A B V_{i}}=0
$$

where $A_{A B V_{i}}$ is the area of the triangle between the vertices $A, B$ and $V_{i}$, and $\epsilon_{A B V_{i}}$ is the deficit angle at the corresponding triangle. Due to our symmetric setting, neither of these depend on $i$, and is solved if either of the following equations holds:

$$
\begin{aligned}
\frac{\partial A_{A B V_{i}}}{\partial t} & =\frac{t\left(a^{2}+b^{2}-t^{2}\right)}{2 \sqrt{-t^{4}-\left(a^{2}-b^{2}\right)^{2}+2 t^{2}\left(a^{2}+b^{2}\right)}}=0 \\
\epsilon_{A B V_{i}} & =2 \pi-3 \arccos \left[\frac{-\left(a^{2}-b^{2}\right)^{2}+2\left(a^{2}+b^{2}-1\right) t^{2}-t^{4}}{2\left(a^{4}+t^{2}+\left(b^{2}-t^{2}\right)^{2}-2 a^{2}\left(b^{2}+t^{2}\right)\right)}\right]=0 .
\end{aligned}
$$

The equation (A.2) has $t=t_{\perp}:=\sqrt{a^{2}+b^{2}}$ as a solution. The only two positive solutions for the second equation are

$$
t=t_{ \pm}:=\frac{1}{2} \sqrt{4 a^{2}+4 b^{2}-3 \pm \sqrt{8 a^{2}-3} \sqrt{8 b^{2}-3}}
$$

The two latter solutions $t_{ \pm}$can readily be identified as the two flat solutions pointing in 'forward' and 'backward' direction. (For $b>a$ the solution $t_{-}$is forward pointing, whereas $t_{+}$is backward pointing.)

\section{A.2 Two tent moves}

We now again start with the three-dimensional triangulation shown in figure A.1, but successively apply two tent moves to the vertex $A$. The resulting triangulation has an inner vertex, $B$, connected to the vertices $V_{i}$, the length denoted by $b$. The final spatial triangulation contains a vertex $C$, the lengths of the edges connecting any of the $V_{i}$ to $C$ being of length $c$. For simplicity, we choose $c=a$ for the moment. Denote the lengths of the tent pole connecting $A$ and $B$, and $B$ and $C$ by $s_{1}$ and $s_{2}$ respectively. Due to symmetry, the Regge equations for $s_{1}, s_{2}$ can immediately be solved to be either of

$$
s_{1,2}=t_{\perp}, t_{ \pm}
$$


with $t_{\perp}:=\sqrt{a^{2}+b^{2}}$ and $t_{ \pm}$given by (A.4). Note that there is still one more equation to solve, which is the equation for $b$, the bulk edge connecting $V_{i}$ and $B$. The Regge equation for $b$ reads

$$
-\frac{\partial S}{\partial b}=\frac{\partial A_{A B V_{i}}}{\partial b} \epsilon_{A B V_{i}}+\frac{\partial A_{C B V_{i}}}{\partial b} \epsilon_{C B V_{i}}+3 \frac{\partial A_{B V_{i} V_{j}}}{\partial b} \epsilon_{B V_{i} V_{j}}=0
$$

The equation (A.6) will have a different form for each of the six choices (A.5) for the lengths $s_{1}, s_{2}$, and result in different equations for $b$.

We first consider the cases where $s_{1}, s_{2}=t_{ \pm}$. As we have already seen, this results in the deficit angles $\epsilon_{A B V_{i}}=\epsilon_{C B V_{i}}$ to vanish. Since for any $i \neq j$ one has

$$
\frac{\partial A_{B V_{i} V_{j}}}{\partial b}=\frac{b}{2 \sqrt{4 b^{2}-1}}
$$

the only way (nonzero b) for (A.6) to be satisfied is that also $\epsilon_{B V_{i} V_{j}}=0$, so the overall triangulation is flat.

It is now not hard to show - in case that either $s_{1}=t_{+}, s_{2}=t_{-}$or $s_{1}=t_{-}, s_{2}=t_{+}-$that $\epsilon_{B V_{i} V_{j}}$ is identically zero for $b$ lying in some finite interval (which, after some investigation, can be found to be the range of values such that all triangles in the triangulation satisfy the triangle inequalities). This gives a one parameter family of flat solutions for the two cases. In the case that either $s_{1}=s_{2}=t_{+}$or $s_{1}=s_{2}=t_{-}$, the only positive solution is

$$
b=\sqrt{\frac{3}{8}}
$$

which is the point where $t_{+}=t_{-}$, so this is just a special case of the flat solution.

Next we consider $s_{1}=t_{+}$and $s_{2}=t_{\perp}$. Again, one finds that the deficit angle $\epsilon_{B V_{i} V_{j}}$ has to vanish, resulting in a completely flat triangulation. The only positive solution is

$$
b=\frac{\sqrt{3} a}{\sqrt{8 a^{2}-3}}
$$

being the solution of $t_{\perp}=t_{-}$, hence again a special case of $s_{1}=t_{+}$and $s_{2}=t_{-}$. The case $s_{1}=t_{-}$and $s_{2}=t_{\perp}$ works analogously.

There is one case left, which is $s_{1}=s_{2}=t_{\perp}=\sqrt{a^{2}+b^{2}}$. Since neither of the deficit angles has to vanish now, the Regge equation (A.6) involves multiple terms, therefore is genuinely a transcendental equation, given by

$$
a\left(2 \pi-3 \arccos \frac{-b^{2}+a^{2}\left(2 b^{2}-1\right)}{b^{2}+a^{2}\left(4 b^{2}-1\right)}\right)+\frac{3 b}{2 \sqrt{4 b^{2}-1}}\left(2 \pi-4 \arccos \frac{b^{2}}{\sqrt{\left(3 b^{2}-1\right)\left(-b^{2}+a^{2}\left(4 b^{2}-1\right)\right)}}\right)=0 .
$$

It still can be solved numerically. For $a=4$, one can plot the derivative $-\partial S / \partial b$ w.r.t $b$, see Figure 12,

The zero can be evaluated numerically to be $b \approx 0.604458$. The deficit angles can be computed numerically for this case as well, and are all found to be unequal to zero:

$$
\epsilon_{A B V_{i}}=\epsilon_{C B V_{i}} \approx-0.615334, \quad \epsilon_{B V_{i} V_{j}} \approx 1.84412 .
$$

The four-dimensional volume of the triangulation is computed to be

$$
V=8 V_{A B V_{i} V_{j} V_{k}} \approx 0.180461
$$

Similarly, the three-dimensional volumes of the tetrahedra can be computed and found to be positive, as well as all areas of triangles. Therefore, all generalized triangle inequalities are satisfied and the solution defines a geometrical triangulation. 


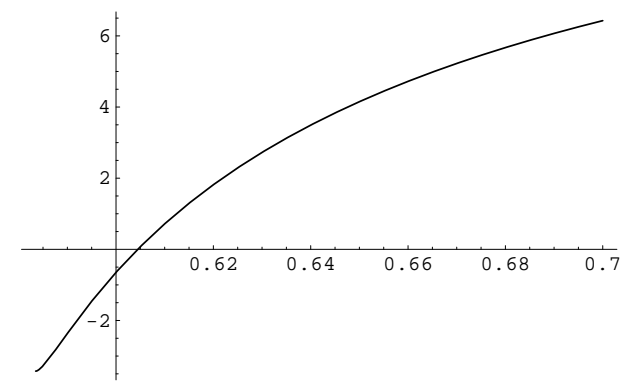

Figure 12: The derivative $-\partial S / \partial b$ as a function of $b$.

Note that - with the same boundary conditions $a=c=4$, there could always be chosen the flat solution $s_{1}=t_{+}, s_{2}=t_{-}$, e.g. with $b=\sqrt{3 / 8}$, for definiteness. The corresponding volume can be computed to be

$$
V=8 V_{A B V_{i} V_{j} V_{k}} \approx 0.232924
$$

which is different from the curved case.

This gives an explicit example where the the specification of boundary values7, and a fixed triangulation (containing an inner vertex) does not result in a unique solution. Apart from the flat solution, there is a curved one as well. Also, this curved solution is isolated, i.e. there is no continuous symmetry of the solution, which we now confirm by computing the Hessian of the action at the solution.

In our triangulation there are six edges meeting at the vertex $B$. Therefore, the Hessian is a

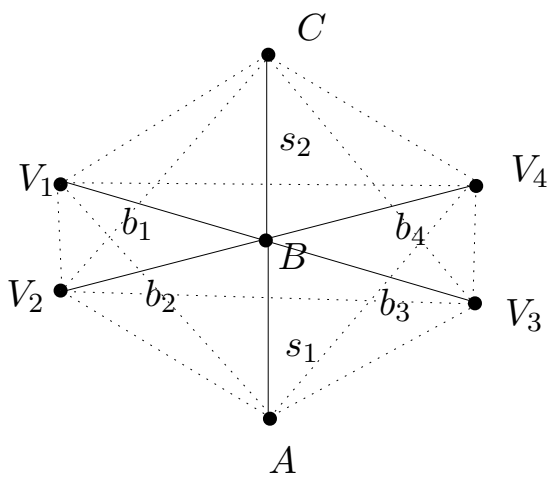

Figure 13: The $4 \mathrm{~d}$ triangulation with inner vertex $B$.

$6 \times 6$ matrix the entries being the second derivatives of the action. Since our solution is highly symmetric, with $b_{1}=\ldots, b_{4}=b$ and $s_{1}=s_{2}$, some of the eigenvalues coincide

$$
\begin{aligned}
& \lambda_{1} \approx-137.752, \quad \lambda_{2} \approx-8.32992, \quad \lambda_{3} \approx-8.32393 \\
& \lambda_{4}=\lambda_{5}=\lambda_{6} \approx 0.339397 .
\end{aligned}
$$

One can see that the Hessian possesses no zero eigenvalue, showing that there is no infinitesimal symmetry of this solution. It is instructive to compare this solution with the flat, homogenous

\footnotetext{
${ }^{7}$ In this case we chose $a=c=4$ for simplicity, however the same type of curved solution exist also for $a \neq c$.
} 
solution, which is given by

$$
b=\sqrt{\frac{3}{8}}, s_{1}=s_{2}=\sqrt{a^{2}-\frac{3}{8}} .
$$

In this case there are four null eigenvectors that can be associated to translations of the inner vertex in the four directions.

\section{B Geometric relations in simplices}

Consider a $D$-dimensional simplex in $D$-dimensional Euclidean space or in $D$-dimensional manifold of constant sectional curvature $\kappa \neq 0$ consisting of $D+1$ vertices $v_{1}, \ldots, v_{D+1}$. Denote this simplex by $(123 \ldots D+1)$. Any subsimplex is determined by the subset $v_{i_{1}}, \ldots, v_{i_{n}}$ of the vertices which span this subsimplex, and will therefore be denoted as $\left(i_{1} i_{2}, \ldots, i_{n}\right) \cdot 8$ Similarly, the notion $\left(\hat{i}_{1} \hat{i}_{2} \ldots, \hat{i}_{n}\right)$ is denoting the subsimplex which consists of all vertices other than $i_{1}, \ldots, i_{n}$. The subsimplices in curved space are defined to be the hypersurfaces with zero extrinsic curvature as embeddings in the geometry of the higher dimensional simplex. These are in fact also simplices of curvature $\kappa$. An edge $(i j)$ is then just given by the geodesic connecting $v_{i}$ and $v_{j} 9$

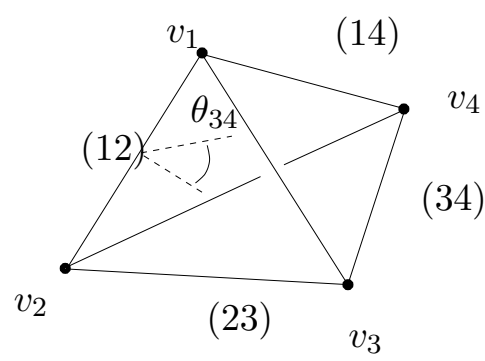

Figure 14: The interior dihedral angle in the tetrahedron (1234) at the edge $(\hat{3} \hat{4})=(12)$.

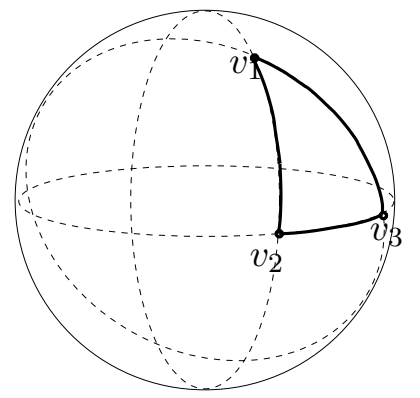

Figure 15: A spherical triangle in $S^{2}$ is a simplex of constant curvature 1 . Note that the edges are simplices in $S^{1}$, which are the great circles, having zero extrinsic curvature in $S^{2}$.

Denote the geodesic lengths of the edges $(i j)$ by $l_{i j}$. Then the $(D+1) \times(D+1)$ matrix $G$ with entries

$$
G_{i j}=c_{\kappa}\left(l_{i j}\right)
$$

where the function $c_{\kappa}(x)$ is defined by

$$
c_{\kappa}(x):= \begin{cases}\cos (\sqrt{\kappa} x) & \kappa>0 \\ \cosh (\sqrt{-\kappa} x) & \kappa<0\end{cases}
$$

is called the Gram matrix of the simplex. For $\kappa=0$ we define

$$
G_{i j}=-\frac{1}{2} \sum_{k, l} l_{k l}^{2}\left(\delta_{i k}-\frac{1}{D+1}\right)\left(\delta_{j l}-\frac{1}{D+1}\right) .
$$

\footnotetext{
${ }^{8}$ The order of the $i_{k}$ determines also an orientation of the subsimplex, which however plays a minor rôle in this article.

${ }^{9}$ Note that if $\kappa>0$ then there are at least two such geodesics. In fact, there are several different curved simplices which have the same vertices. For definiteness we will always choose the shorter of the two geodesics, and not consider cases in which there are infinitely many such geodesics, which are degenerate anyway.
} 
to be the affine metric of the simplex, see for instance [23]. For $\kappa \neq 0$ we denote by $c_{i j}$ the $i j$-th cofactor of $G$, i.e. the determinant of the matrix obtained by removing the $i$-th row and $j$-th column of $G$. For the flat case $\kappa=0$, we take $c_{i j}$ to be the affine inverse metric, that is $\sum_{k} G_{i k} c_{k j}=\delta_{i j}-\frac{1}{D+1}$.

Then the interior dihedral angle $\theta_{[i j]}$ opposite of the edge $(i j)$ is given by [37, 23]

$$
\cos \theta_{[i j]}=-\frac{c_{i j}}{\sqrt{c_{i i}} \sqrt{c_{j j}}}
$$

Denote the volume of the subsimplex spanned by all vertices except $v_{i}$ and $v_{j}$ by $V_{(\hat{i} \hat{j})}$. For variations $\delta$ of the geometry of a simplex the Schläfli-identity [38]

$$
\sum_{i<j} V_{(\hat{i} \hat{j})} \delta \theta_{[i j]}=(D-1) \kappa \delta V_{(12 \ldots D+1)}
$$

holds.

With the help of this identity we can derive some formulas needed in section 6. First of all if for a given tetrahedron we define the function

$$
S_{\tau}=\sum_{i<j} V_{(\hat{i} \hat{j})} \theta_{[i j]}-2 \kappa V_{(12 \ldots D+1)}
$$

we can use it as a generating function for the dihedral angles

$$
\theta_{[i j]}=\frac{\partial S_{\tau}}{\partial V_{(\hat{i} \hat{j})}}
$$

where $V_{(\hat{i} \hat{j})}$ is the length of the edge between the two other vertices $k, l \neq i, j$ of the tetrahedron. Since partial derivatives commute we can conclude

$$
\frac{\partial \theta_{[i j]}}{\partial V_{(\hat{k} \hat{l})}}=\frac{\partial \theta_{[k l]}}{\partial V_{(\hat{i} \hat{j})}}
$$

which is used in section 6, for instance to see that the constraints derived there are Abelian.

To compute the derivative of the dihedral angles with respect to the sectional curvature $\kappa$, also needed in section 6, we note from the definition (B.2[B.3) of the dihedral angles that

$$
\begin{aligned}
\frac{\mathbf{d} \theta_{[k l]}}{\mathbf{d} \kappa} & =\sum_{i>j} \frac{\partial \theta_{[k l]}}{\partial V_{(\hat{i} \hat{j})}} \frac{1}{2} \kappa^{-1} V_{(\hat{i} \hat{j})} \\
& =\sum_{i>j} \frac{\partial \theta_{[i j]}}{\partial V_{(\hat{k} \hat{l})}} \frac{1}{2} \kappa^{-1} V_{(\hat{i} \hat{j})} \\
& =\frac{\partial V_{(1234)}}{\partial V_{(\hat{k} \hat{l})}}
\end{aligned}
$$

where in the second line we use (B.7) and in the third line the Schlälfi identity (B.4).

The derivative of the dihedral angles can be also computed from their definition (B.2] B.3

$$
\frac{\partial \theta_{[k l]}}{\partial l_{o p}}=-\frac{1}{\sin \theta_{[k l]}} \frac{1}{\sqrt{c_{k k} c_{l l}}} \frac{1}{2} \sum_{h, m}\left(c_{k h} c_{m l}+c_{k m} c_{h l}-\frac{c_{k l}}{c_{k k}} c_{k h} c_{k m}-\frac{c_{k l}}{c_{l l}} c_{l h} c_{l m}\right) \frac{\partial G_{h m}}{\partial l_{o p}},
$$

see also [23] for simplifications in the Euclidean case. 
Finally the volume of an Euclidean $D$-simplex can be computed by using a generalization of Heron's formula

$$
V_{(12 \ldots D+1)}^{2}=(-1)^{D+1} \frac{1}{2^{D}(D !)^{2}} \operatorname{det}\left(\begin{array}{ccccc}
0 & 1 & 1 & \cdots & 1 \\
1 & 0 & l_{12}^{2} & \cdots & l_{1 D+1}^{2} \\
1 & l_{12}^{2} & 0 & \cdots & l_{2 D+1}^{2} \\
\vdots & & & \ddots & \\
1 & l_{1 D+1}^{2} & l_{2 D+1}^{2} & \cdots & l_{D+1 D+1}^{2}
\end{array}\right)
$$

and one can show that for the derivative of this volume we have

$$
\cos \theta_{[i j]}=\frac{D^{2}}{V_{(\hat{i})} V_{(\hat{j})}} \frac{\partial V_{(12 \ldots D+1)}^{2}}{\partial\left(l_{i j}^{2}\right)}=\frac{D^{2}}{2 l_{i j} V_{(\hat{i})} V_{(\hat{j})}} \frac{\partial V_{(12 \ldots D+1)}^{2}}{\partial l_{i j}} .
$$

\section{Acknowledgements}

It is a pleasure to thank John Barrett, Philipp Höhn, Renate Loll and Ruth Williams for discussions. The research of B.D. at the University of Utrecht was supported by a Marie Curie Fellowship of the European Union.

\section{References}

[1] C. Fleischhack, "Representations of the Weyl Algebra in Quantum Geometry," to appear in Commun. Math. Phys. arXiv:math-ph/0407006, J. Lewandowski, A. Okolow, H. Sahlmann and T. Thiemann, "Uniqueness of diffeomorphism invariant states on holonomy-flux algebras," Commun. Math. Phys. 267 (2006) 703 arXiv:gr-qc/0504147.

[2] T. Regge, "General relativity without coordinates," Nuovo Cim. 19 (1961) 558.

[3] T. Regge and R. M. Williams, "Discrete structures in gravity," J. Math. Phys. 41 (2000) 3964 arXiv:gr-qc/0012035.

[4] A. Perez, "Spin foam models for quantum gravity," Class. Quant. Grav. 20 (2003) R43 arXiv:gr-qc/0301113]. "Introduction to loop quantum gravity and spin foams," arXiv:gr-qc/0409061. J. Engle, R. Pereira and C. Rovelli, "The loop-quantum-gravity vertex-amplitude," Phys. Rev. Lett. 99 (2007) 161301 arXiv:0705.2388 [gr-qc]]. E. R. Livine and S. Speziale, "Consistently Solving the Simplicity Constraints for Spinfoam Quantum Gravity," Europhys. Lett. 81 (2008) 50004 [arXiv:0708.1915 [gr-qc]]. L. Freidel and K. Krasnov, Class. Quant. Grav. 25 (2008) 125018 [arXiv:0708.1595 [gr-qc]].

[5] J. Ambjorn, J. Jurkiewicz and R. Loll, "Dynamically triangulating Lorentzian quantum gravity," Nucl. Phys. B 610 (2001) 347 [arXiv:hep-th/0105267]. R. Loll, "The Emergence of Spacetime, or, Quantum Gravity on Your Desktop," Class. Quant. Grav. 25 (2008) 114006 [arXiv:0711.0273 [gr-qc]].

[6] F. Conrady and L. Freidel, "Path integral representation of spin foam models of 4d gravity," Class. Quant. Grav. 25 (2008) 245010 arXiv:0806.4640 [gr-qc]]. F. Conrady and L. Freidel, "On the semiclassical limit of 4d spin foam models," Phys. Rev. D 78 (2008) 104023 arXiv:0809.2280 [gr-qc]]. J. W. Barrett, R. J. Dowdall, W. J. Fairbairn, H. Gomes and F. Hellmann, "Asymptotic analysis of the EPRL four-simplex amplitude," arXiv:0902.1170 [gr-qc].

[7] T. Piran and R. M. Williams, "A $(3+1)$ Formulation Of Regge Calculus," Phys. Rev. D 33 (1986) 1622. J. L. Friedman and I. Jack, "(3+1) Regge Calculus With Conserved Momentum And Hamiltonian Constraints," J. Math. Phys. 27 (1986) 2973. 
[8] R. Loll, "On the diffeomorphism-commutators of lattice quantum gravity," Class. Quant. Grav. 15 (1998) 799 arXiv:gr-qc/9708025].

[9] H. W. Hamber and R. M. Williams, "Gauge invariance in simplicial gravity," Nucl. Phys. B 487 (1997) 345 arXiv:hep-th/9607153.

[10] J. B. Hartle, "Simplicial Minisuperspace. I. General Discussion," J. Math. Phys. 26 (1985) 804. W. A. Miller, "The geometrodynamic content of the Regge equations as illuminated by the boundary of a boundary principle," Found. Phys. 16 (1986) 143. A. P. Gentle, A. Kheyfets, J. R. McDonald and W. A. Miller, "A Kirchoff-like conservation law in Regge calculus," arXiv:0807.3041 [gr-qc].

[11] P. A. Morse, "Approximate diffeomorphism invariance in near flat simplicial geometries," Class. Quant. Grav. 9 (1992) 2489.

[12] M. Galassi, "Lapse and shift in Regge calculus," Phys. Rev. D 47 (1993) 3254. A. P. Gentle and W. A. Miller, "A fully (3+1)-D Regge calculus model of the Kasner cosmology," Class. Quant. Grav. 15 (1998) 389 arXiv:gr-qc/9706034.

[13] H. Waelbroeck and J. A. Zapata, "A Hamiltonian formulation of topological gravity," Class. Quant. Grav. 11 (1994) 989 arXiv:gr-qc/9311035. J. A. Zapata, "Topological Lattice Gravity Using Self-Dual Variables," Class. Quant. Grav. 13 (1996) 2617 arXiv:gr-qc/9603030.

[14] B. Dittrich and J. P. Ryan, "Phase space descriptions for simplicial 4d geometries," arXiv:0807.2806 [gr-qc].

[15] J. R. Klauder, "Coherent state quantization of constraint systems," Annals Phys. 254 (1997) 419 arXiv:quant-ph/9604033. "Universal procedure for enforcing quantum constraints," Nucl. Phys. B 547 (1999) 397 arXiv:hep-th/9901010. "Quantization of constrained systems," Lect. Notes Phys. 572 (2001) 143 [arXiv:hep-th/0003297].

[16] T. Thiemann, "The Phoenix project: Master constraint programme for loop quantum gravity," Class. Quant. Grav. 23 (2006) 2211 arXiv:gr-qc/0305080. B. Dittrich and T. Thiemann, "Testing the master constraint programme for loop quantum gravity. I: General framework," Class. Quant. Grav. 23 (2006) 1025 arXiv:gr-qc/0411138. II: Finite dimensional systems," Class. Quant. Grav. 23 (2006) 1067 [arXiv:gr-qc/0411139]. III: SL(2,R) models," Class. Quant. Grav. 23 (2006) 1089 arXiv:gr-qc/0411140|. "Testing the master constraint programme for loop quantum gravity. IV: Free field theories," Class. Quant. Grav. 23 (2006) 1121 arXiv:gr-qc/0411141]. V: Interacting field theories," Class. Quant. Grav. 23 (2006) 1143 arXiv:gr-qc/0411142.

[17] M. Campiglia, C. Di Bartolo, R. Gambini and J. Pullin, "Uniform discretizations: A new approach for the quantization of totally constrained systems," Phys. Rev. D 74 (2006) 124012 arXiv:gr-qc/0610023.

[18] R. Gambini and J. Pullin, "Canonical quantization of general relativity in discrete spacetimes," Phys. Rev. Lett. 90 (2003) 021301 arXiv:gr-qc/0206055. C. Di Bartolo, R. Gambini, R. Porto and J. Pullin, "Dirac-like approach for consistent discretizations of classical constrained theories," J. Math. Phys. 46 (2005) 012901 arXiv:gr-qc/0405131.

[19] J. J. Halliwell and J. B. Hartle, "Wave functions constructed from an invariant sum over histories satisfy constraints," Phys. Rev. D 43 (1991) 1170.

[20] B. Dittrich, "Diffeomorphism symmetry in quantum gravity models," Adv. Sci. Lett. 2 (2209) 121132 arXiv:0810.3594 [gr-qc]].

[21] M. Rocek and R. M. Williams, "Quantum Regge Calculus," Phys. Lett. B 104 (1981) 31. "The Quantization Of Regge Calculus," Z. Phys. C 21 (1984) 371.

[22] L. Freidel and D. Louapre, "Diffeomorphisms and spin foam models," Nucl. Phys. B 662 (2003) 279 arXiv:gr-qc/0212001.

[23] B. Dittrich, L. Freidel and S. Speziale, "Linearized dynamics from the 4-simplex Regge action," Phys. Rev. D 76 (2007) 104020 [arXiv:0707.4513 [gr-qc]]. 
[24] R. Sorkin, "Time Evolution Problem In Regge Calculus," Phys. Rev. D 12 (1975) 385 [Erratum-ibid. D 23 (1981) 565]. P. A. Tuckey, "The Construction of Sorkin triangulations," Class. Quant. Grav. 10 (1993) L109 arXiv:gr-qc/9303011. J. W. Barrett, M. Galassi, W. A. Miller, R. D. Sorkin, P. A. Tuckey and R. M. Williams, "A Paralellizable implicit evolution scheme for Regge calculus," Int. J. Theor. Phys. 36 (1997) 815 arXiv:gr-qc/9411008.

[25] J. Marsden, M. West, "Discrete mechanics and variational integrators," Acta Numerica 10 (2001) 357

[26] J. B. Hartle,R. Sorkin, "Boundary terms in the action for the Regge calculus," Gen. Rel. Grav. 13 (1981), 541

[27] T. Piran and A. Strominger, "Solutions to the Regge equations," Class. Quant. Grav. 3 (1986) 97.

[28] B. Bahr, B. Dittrich, P. Höhn, "Exact and approximate constraints in 4d Regge calculus," to appear

[29] R. Gambini and J. Pullin, "Consistent discretization and canonical classical and quantum Regge calculus," Int. J. Mod. Phys. D 15 (2006) 1699 [arXiv:gr-qc/0511096].

[30] B. Bahr, B. Dittrich, "Improving the action for Regge calculus with cosmological constant," to appear

[31] B. Dittrich and L. Freidel, to appear

[32] J. F. Plebanski, "On the separation of Einsteinian substructures," J. Math. Phys. 18 (1977) 2511.

[33] B. Dittrich and S. Speziale, "Area-angle variables for general relativity," New J. Phys. 10 (2008) 083006 arXiv:0802.0864 [gr-qc]].

[34] M. Lehto, H. B. Nielsen and M. Ninomiya, "Diffeomorphism Symmetry In Simplicial Quantum Gravity," Nucl. Phys. B 272 (1986) 228.

[35] P. Hasenfratz, "The theoretical background and properties of perfect actions," arXiv:hep-lat/9803027.

[36] D. Oriti, "The group field theory approach to quantum gravity," arXiv:gr-qc/0607032.

[37] S. L. Kokkendorff, "Polar Duality and the Generalized Law of Sines," Journal of Geometry 86 (2007) 140

[38] J. Milnor, Collected papres. Vol 1, Publish or Persih Inc., Houston, TX, 1994 